\title{
Shape derivatives of boundary integral oper- ators in electromagnetic scattering. Part I: Shape differentiability of pseudo-homogene- ous boundary integral operators
}

\author{
Martin Costabel and Frédérique Le Louër
}

\begin{abstract}
In this paper we study the shape differentiability properties of a class of boundary integral operators and of potentials with weakly singular pseudo-homogeneous kernels acting between classical Sobolev spaces, with respect to smooth deformations of the boundary. We prove that the boundary integral operators are infinitely differentiable without loss of regularity. The potential operators are infinitely shape differentiable away from the boundary, whereas their derivatives lose regularity near the boundary. We study the shape differentiability of surface differential operators. The shape differentiability properties of the usual strongly singular or hypersingular boundary integral operators of interest in acoustic, elastodynamic or electromagnetic potential theory can then be established by expressing them in terms of integral operators with weakly singular kernels and of surface differential operators.
\end{abstract}

Keywords. Boundary integral operators, pseudo-homogeneous kernels, fundamental solution, surface differential operators, shape derivatives, Sobolev spaces.

\section{Introduction}

Optimal shape design problems and inverse problems involving the scattering of time-harmonic waves are of practical interest in many important fields of applied physics including radar and sonar applications, structural design, biomedical imaging and non destructive testing. We develop new analytic tools that can be used in algorithms for the numerical solution of such problems.

Shape derivatives are a classical tool in shape optimization and are also widely used in inverse obstacle scattering. In shape optimization, where extrema of cost functions have to be determined, the analysis of iterative 
methods requires the study of the derivative of the solution of a scattering problem with respect to the shape of the boundary of the obstacle. An explicit form of the shape derivatives is required in view of their implementation in iterative algorithms such as gradient methods or Newton's method [5, 9, 24. By the method of boundary integral equations, the shape analysis of the solution of the scattering problem with respect to deformations of the obstacle is obtained from the Gâteaux differentiability analysis of boundary integral operators and potentials with weakly singular, strongly singular, or hypersingular kernels. An expression of the shape derivatives of the solution can then be computed by taking the derivative of its integral representation. This technique was introduced for the Dirichlet and Neumann problems in acoustic scattering by Potthast [21, 22, and applied to the Dirichlet problem in elastic scattering by Charalambopoulos [1] in the framework of Hölder continuous and differentiable function spaces. More recently these results were exploited in acoustic inverse obstacle scattering to develop novel methods in which a system of nonlinear integral equations has to be solved by a regularized iterative method [15, 13, 12.

An extension of the technique to elasticity and electromagnetism requires the shape differentiability analysis of the relevant boundary integral operators. More generally, we are concerned in this paper with the Gâteaux differentiability of boundary integral operators with strongly and weakly singular pseudo-homogeneous kernels acting between classical Sobolev spaces, with respect to smooth deformations of the boundary considered as a hypersurface of $\mathbb{R}^{d}$ with $d \in \mathbb{N}, d \geq 2$. This family of integral operators covers the case of the single and double layer integral operators from the acoustic and the elastic scattering potential theory. The differentiability properties of the hypersingular boundary integral operators can then be established by expressing them as products of integral operators with weakly singular kernels and of surface differential operators. In return, however, we have to study the shape differentiability of surface differential operators. The electromagnetic case presents a specific difficulty: The associated boundary integral operators act as bounded operators on the space of tangential vector fields of mixed regularity $\mathbf{T H}^{-\frac{1}{2}}\left(\operatorname{div}_{\Gamma}, \Gamma\right)$. The very definition of the shape derivative of an operator defined on this energy space poses non-trivial problems. This is the subject of the second part of this paper [3] where we propose an analysis based on the Helmholtz decomposition 4 of $\mathbf{T H}^{-\frac{1}{2}}\left(\operatorname{div}_{\Gamma}, \Gamma\right)$.

This work contains results from the thesis [17] where this analysis has been used to construct and to implement shape optimization algorithms for dielectric lenses, aimed at obtaining a prescribed radiation pattern.

The paper is organized as follows:

In Section 2 we describe the family of pseudo-differential boundary integral operators and potentials that we consider. We use a subclass of the class of pseudo-homogeneous kernels introduced by Nédélec in his book 20]. Main results on the regularity of these operators are set out. In Section 3 , we define the notion of shape derivative and discuss its connection to Gâteaux 
derivatives. We also recall elementary results about differentiability in Fréchet spaces, following ideas of [5, 6] and notations of [23].

Section 4 is dedicated to the shape differentiability analysis of the integral operators. We discuss different definitions of derivatives with respect to deformations of the boundary and compare them to the notions of material derivatives and shape derivatives that are common in continuum mechanics, see Remark 4.1. We prove that shape derivatives of the boundary integral operators are operators of the same class, that the boundary integral operators are infinitely shape differentiable without loss of regularity, and that the potentials are infinitely shape differentiable away from the boundary of the obstacle, whereas their derivatives lose regularity in the neighborhood of the boundary. A main tool is the proof that the shape differentiability of the integral operators can be reduced to the one of their kernels. We also give higher order Gâteaux derivatives of coefficient functions such as the Jacobian of the change of variables associated with the deformation, or the components of the unit normal vector. These results are new and allow us to obtain explicit forms of higher order derivatives of the integral operators. A utilization for the implementation of higher order iterative methods is conceivable.

The shape differentiability properties of usual surface differential operators is given in the last section. Again we prove their infinite Gâteaux differentiability and give an explicit expression of their derivatives. These are then applied to obtain the derivatives of hypersingular boundary integral operators from acoustic, elastic and electromagnetic potential theory.

Notice that our shape differentiability analysis is realized without restriction to particular classes of deformations of the boundary, such as it is frequently done in the calculus of variations, namely restriction to deformations normal to the surface as suggested by the structure theorems for shape derivatives [8, 9, 24], or consideration of radial deformations of star-shaped surfaces [2, 13, 12].

\section{Pseudo-homogeneous kernels}

Let $\Omega$ denote a bounded domain in $\mathbb{R}^{d}$ with $d \geq 2$ and let $\Omega^{c}$ denote the exterior domain $\mathbb{R}^{d} \backslash \bar{\Omega}$. In this paper, we will assume that the boundary $\Gamma$ of $\Omega$ is a smooth closed hypersurface. Let $\boldsymbol{n}$ denote the outer unit normal vector on $\Gamma$.

For a domain $G \subset \mathbb{R}^{d}$ we denote by $H^{s}(G)$ the usual $L^{2}$-based Sobolev space of order $s \in \mathbb{R}$, and by $H_{\mathrm{loc}}^{s}(\bar{G})$ the space of functions whose restrictions to any bounded subdomain $B$ of $G$ belong to $H^{s}(B)$.

For any $t \in \mathbb{R}$ we denote by $H^{t}(\Gamma)$ the standard Sobolev space on the boundary $\Gamma$. The dual of $H^{t}(\Gamma)$ with respect to the $L^{2}$ scalar product is $H^{-t}(\Gamma)$. Vector functions and spaces of vector functions will be denoted by boldface letters. 
For $\alpha=\left(\alpha_{1}, \ldots, \alpha_{d}\right) \in \mathbb{N}^{d}$ and $z=\left(z_{1}, \ldots, z_{d}\right) \in \mathbb{R}^{d}$ we denote by $\frac{\partial^{|\alpha|}}{\partial z^{\alpha}}$ the linear partial differential operator defined by

$$
\frac{\partial^{|\alpha|}}{\partial z^{\alpha}}=\frac{\partial^{\alpha_{1}}}{\partial z_{1}^{\alpha_{1}}} \cdots \frac{\partial^{\alpha_{d}}}{\partial z_{d}^{\alpha_{d}}}
$$

where $|\alpha|=\alpha_{1}+\cdots+\alpha_{d}$. For $m \in \mathbb{N}$, the total differential of order $m$, a symmetric $m$-linear form on $\mathbb{R}^{d}$, is denoted by $\mathrm{D}^{m}$.

The integral operators we consider can be written in the form

$$
\mathcal{K}_{\Gamma} u(x)=\int_{\Gamma} k(y, x-y) u(y) d s(y), \quad x \in \Gamma,
$$

where the integral is assumed to exist in the sense of a Cauchy principal value and the kernel $k$ is regular with respect to the variable $y \in \Gamma$ and pseudohomogeneous with respect to the variable $z=x-y \in \mathbb{R}^{d}$. We recall the regularity properties of these operators on the Sobolev spaces $H^{t}(\Gamma)$ for all $t \in \mathbb{R}$, available also for their adjoint operators

$$
\mathcal{K}_{\Gamma}^{*}(u)(x)=\int_{\Gamma} k(x, y-x) u(y) d s(y), x \in \Gamma .
$$

We use a variant of the class of weakly singular kernels introduced by Nédélec in [20, pp. 168ff]. More details can be found in [7, 10, 14, 19, 26, 25].

Definition 2.1. The kernel $G(z) \in \mathscr{C}^{\infty}\left(\mathbb{R}^{d} \backslash\{0\}\right)$ is said to be homogeneous of class $-m$ for an integer $m \geq 0$ if

(i) for any $\alpha \in \mathbb{N}^{d}$ there is a constant $C_{\alpha}$ such that for all $z \in \mathbb{R}^{d} \backslash\{0\}$

we have $\quad\left|\frac{\partial^{|\alpha|}}{\partial z^{\alpha}} G(z)\right| \leq C_{\alpha}|z|^{-(d-1)+m-|\alpha|}$,

(ii) for any $\alpha \in \mathbb{N}^{d}$ with $|\alpha|=m$, the function $\frac{\partial^{|\alpha|}}{\partial z^{\alpha}} G(z)$ is homogeneous of degree $-(d-1)$ with respect to the variable $z$,

(iii) $\mathrm{D}^{m} G(z)$ is an odd function of $z$.

Remark 2.2. (i) The number $-m$ in this definition is not the order of homogeneity of the kernel, but related to the order of the corresponding pseudodifferential operator defined on the $d$-1-dimensional manifold $\Gamma$.

(ii) Our condition (iii) is stronger than the vanishing condition in Nedelec's original definition, but it is easier to verify, and it is satisfied for the classical integral operators we will be considering.

Definition 2.3. The kernel $k(y, z)$ defined on $\Gamma \times\left(\mathbb{R}^{d} \backslash\{0\}\right)$ is said to be pseudo-homogeneous of class $-m$ for an integer $m$ such that $m \geq 0$, if the kernel $k$ admits the following asymptotic expansion when $z$ tends to 0 :

$$
k(y, z)=\sum_{j \geq 0, \ell} b_{m+j}^{\ell}(y) G_{m+j}^{\ell}(z),
$$


where for $j=0,1, \ldots$ the sum over $\ell$ is finite, $b_{m+j}^{\ell}$ belongs to $\mathscr{C}^{\infty}(\Gamma)$ and $G_{m+j}^{\ell}$ is homogeneous of class $-(m+j)$.

In (2.3), one can also consider coefficient functions of the form $b_{m+j}(x, y)$ with $x=y+z$, but using Taylor expansion of such coefficients at $z=0$, we see that this would define the same class of kernels as with (2.3).

Example 2.4. (Acoustic kernels) Let $\kappa \in \mathbb{C} \backslash\{0\}$ with $\operatorname{Im}(\kappa) \geq 0$ and $d=2$ or $d=3$. The fundamental solution

$$
G_{a}(\kappa, z)= \begin{cases}\frac{i}{4} H_{0}^{(1)}(\kappa|z|) & \text { when } d=2 \\ \frac{e^{i \kappa|z|}}{4 \pi|z|} & \text { when } d=3\end{cases}
$$

of the Helmholtz equation $\Delta u+\kappa^{2} u=0$ in $\mathbb{R}^{d}$ is pseudo-homogeneous of class -1 . Its normal derivative $\frac{\partial}{\partial \boldsymbol{n}(y)} G_{a}(\kappa, z)$ is a priori pseudo-homogeneous of class 0 but one can show that in the case of smooth boundaries it is a pseudo-homogeneous kernel of class -1 .

Indeed one can write

$$
\frac{e^{i \kappa|z|}}{4 \pi|z|}=\frac{1}{|z|}+i \kappa-\frac{\kappa^{2}}{2}|z|-\frac{i \kappa^{3}}{6}|z|^{2}+\ldots
$$

The first term is homogeneous of class -1 , the second term is smooth and for $j \geq 3$ the $j$-th term is homogeneous of class $-(1+j)$. The double layer kernel has the expansion

$$
\frac{\partial}{\partial \boldsymbol{n}(y)} G_{a}(\kappa, z)=\boldsymbol{n}(y) \cdot \nabla^{z} G_{a}(\kappa, z)=(\boldsymbol{n}(y) \cdot z)\left(-\frac{1}{|z|^{3}}-\frac{\kappa^{2}}{2} \frac{1}{|z|}-\frac{i \kappa^{3}}{3}+\ldots\right) \text {. }
$$

One can prove that the function $g(x, y)=\boldsymbol{n}(y) \cdot(x-y)$ behaves as $|x-y|^{2}$ when $z=x-y \rightarrow 0$ (see for instance [20, p. 173]). We refer to example 4.11] for a proof using a local coordinate system.

Example 2.5. (Elastodynamic kernels) Let $\omega \in \mathbb{R}$ and $d=2$ or $d=3$. Denote by $\rho, \mu$ and $\lambda$ the density and Lamé's constants. The symmetric fundamental solution of the Navier equation $-\mu \Delta \boldsymbol{u}-(\mu+\lambda) \nabla \operatorname{div} \boldsymbol{u}-\rho w^{2} \boldsymbol{u}=0$, given by

$$
G_{e}\left(\kappa_{s}, \kappa_{p}, z\right)=\frac{1}{\mu}\left(G_{a}\left(\kappa_{s}, z\right) \cdot \mathrm{I}_{\mathbb{R}^{d}}+\frac{1}{\kappa_{s}^{2}} \operatorname{Hess}\left(G_{a}\left(\kappa_{s}, z\right)-G_{a}\left(\kappa_{p}, z\right)\right)\right),
$$

with $\kappa_{s}=\omega \sqrt{\frac{\rho}{\mu}}$ and $\kappa_{p}=\omega \sqrt{\frac{\rho}{\lambda+2 \mu}}$, is pseudo-homogeneous of class -1 . The traction operator is defined by

$$
T \boldsymbol{u}=2 \mu \frac{\partial \boldsymbol{u}}{\partial \boldsymbol{n}}+\lambda(\operatorname{div} \boldsymbol{u}) \boldsymbol{n}+\mu \boldsymbol{n} \wedge \operatorname{curl} \boldsymbol{u} .
$$

The double layer kernel $\left(T_{y} G_{e}\left(\kappa_{s}, \kappa_{p}, x-y\right)\right)^{\top}$ is pseudo-homogeneous of class 0 . The index $y$ of $T_{y}$ means that the differentiation is with respect to the variable $y$. Notice that $T_{y} G_{e}\left(\kappa_{s}, \kappa_{p}, x-y\right)$ is the tensor obtained by applying the traction operator $T_{y}$ to each column of $G_{e}\left(\kappa_{s}, \kappa_{p}, x-y\right)$. 
For the proof of the following theorem we refer to [20, 25].

Theorem 2.6. Let $k$ be a pseudo-homogeneous kernel of class $-m$. The associated boundary integral operator $\mathcal{K}_{\Gamma}$ given by (2.1) is linear and continuous from $H^{t}(\Gamma)$ to $H^{t+m}(\Gamma)$ for all $t \in \mathbb{R}$. The same result is true for the adjoint operator $\mathcal{K}_{\Gamma}^{*}$.

The following theorem is established in [7].

Theorem 2.7. Let $s \in \mathbb{R}$. Let $k$ be a pseudo-homogeneous kernel of class $-m$. The potential operator $\mathcal{P}$ defined by

$$
\mathcal{P}(u)(x)=\int_{\Gamma} k(y, x-y) u(y) d s(y), \quad x \in \mathbb{R}^{d} \backslash \Gamma
$$

is linear and continuous from $H^{s}(\Gamma)$ to $H^{s+m+\frac{1}{2}}(\Omega) \cup H_{l o c}^{s+m+\frac{1}{2}}\left(\overline{\Omega^{c}}\right)$.

\section{Some remarks on shape derivatives}

We want to study the dependence of operators defined by integrals over the boundary $\Gamma$ on the geometry of $\Gamma$. This dependence is highly nonlinear. The usual tools of differential calculus require the framework of topological vector spaces which are locally convex at least, a framework that is not immediately present in the case of shape functionals. The standard approach consists in representing the variations of the domain $\Omega$ by elements of a function space. We consider variations generated by transformations of the form

$$
x \mapsto x+r(x)
$$

of point $x$ in the space $\mathbb{R}^{d}$, where $r$ is a smooth vector function defined in the neighborhood of $\Gamma$. This transformation deforms the domain $\Omega$ into a domain $\Omega_{r}$ with boundary $\Gamma_{r}$. The functions $r$ are assumed to be sufficiently small elements of the Fréchet space $\mathcal{X}=\mathscr{C}^{\infty}\left(\Gamma, \mathbb{R}^{d}\right)$ in order that $(\mathrm{I}+r)$ is a diffeomorphism from $\Gamma$ to

$$
\Gamma_{r}=(\mathrm{I}+r) \Gamma=\left\{x_{r}=x+r(x) ; x \in \Gamma\right\} .
$$

For $\varepsilon$ small enough we set

$$
B^{\infty}(0, \varepsilon)=\left\{r \in \mathscr{C}^{\infty}\left(\Gamma, \mathbb{R}^{d}\right), d_{\infty}(0, r)<\varepsilon\right\},
$$

where $d_{\infty}$ is the distance induced by the family of non-decreasing norms $\left(\|\cdot\|_{k}\right)_{k \in \mathbb{N}}$ defined by

$$
\|r\|_{k}=\sup _{0 \leq m \leq k} \sup _{x \in \mathbb{R}^{d}}\left|\mathrm{D}^{m} r(x)\right| .
$$

Consider a mapping $F$ defined on the set $\left\{\Gamma_{r} ; r \in B^{\infty}(0, \varepsilon)\right\}$ of boundaries. We introduce a new mapping

$$
B^{\infty}(0, \varepsilon) \ni r \mapsto \mathcal{F}_{\Gamma}(r)=F\left(\Gamma_{r}\right) .
$$


We define the shape derivative of the mapping $F$ through the transformation $\Gamma \ni x \mapsto x+\xi(x) \in \mathbb{R}^{d}$ by

$$
d F[\Gamma ; \xi]:=\lim _{t \rightarrow 0} \frac{F\left(\Gamma_{t \xi}\right)-F(\Gamma)}{t}=\lim _{t \rightarrow 0} \frac{\mathcal{F}_{\Gamma}(t \xi)-\mathcal{F}_{\Gamma}(0)}{t}
$$

if the limit exists and is finite. The shape derivatives of $F$ are related to the Gâteaux derivatives of $\mathcal{F}_{\Gamma}$ (see [9, 24]).

Fix $r_{0} \in B^{\infty}(0, \varepsilon)$. Following the same procedure, one can construct another mapping $\mathcal{F}_{\Gamma_{r_{0}}}$ defined on the family of boundaries

$$
\left\{\left(\mathrm{I}+r^{\prime}\right)\left(\Gamma_{r_{0}}\right) ; r^{\prime} \in B^{\infty}\left(0, \varepsilon^{\prime}\right)\right\} .
$$

Notice that $\mathcal{F}_{\Gamma_{r_{0}}}(0)=F\left(\Gamma_{r_{0}}\right)=\mathcal{F}_{\Gamma}\left(r_{0}\right)$ and $\mathcal{F}_{\Gamma_{r_{0}}}\left(\left(r-r_{0}\right) \circ\left(\mathrm{I}+r_{0}\right)^{-1}\right)=$ $F\left(\Gamma_{r}\right)=\mathcal{F}_{\Gamma}(r)$.

\subsection{Differentiability in Fréchet spaces: elementary results}

Fréchet spaces are locally convex, metrisable and complete topological vector spaces on which the differential calculus available on Banach spaces can be extended. We recall some of the results. We refer to Schwartz's book [23] for more details.

Let $\mathcal{X}$ and $\mathcal{Y}$ be Fréchet spaces and let $U$ be a subset of $\mathcal{X}$.

Definition 3.1. (Gâteaux semi-derivatives) The mapping $f: U \rightarrow \mathcal{Y}$ is said to have a Gâteaux semiderivative at $r_{0} \in U$ in the direction of $\xi \in \mathcal{X}$ if the following limit exists in $\mathcal{Y}$

$$
d f\left[r_{0} ; \xi\right]=\lim _{t \rightarrow 0} \frac{f\left(r_{0}+t \xi\right)-f\left(r_{0}\right)}{t}=\left.\frac{d}{d t}\right|_{t=0} f\left(r_{0}+t \xi\right) .
$$

Definition 3.2. (Gâteaux differentiability) The mapping $f: U \rightarrow \mathcal{Y}$ is said to be Gâteaux differentiable at $r_{0} \in U$ if it has Gâteaux semiderivatives in all directions $\xi \in \mathcal{X}$ and if the mapping

$$
\mathcal{X} \ni \xi \mapsto d f\left[r_{0} ; \xi\right] \in \mathcal{Y}
$$

is linear and continuous.

We say that $f$ is continuously (or $\mathscr{C}^{1}$-) Gâteaux differentiable if it is Gâteaux differentiable at all $r_{0} \in U$ and the mapping

$$
U \times \mathcal{X} \ni d f:\left(r_{0}, \xi\right) \mapsto d f\left[r_{0} ; \xi\right] \in \mathcal{Y}
$$

is continuous.

Remark 3.3. In the calculus of shape derivatives, we usually consider the Gâteaux derivative at $r=0$ only. This is due to the result: If $\mathcal{F}_{\Gamma}$ is Gâteaux differentiable on $B^{\infty}(0, \varepsilon)$, then for all $\xi \in \mathcal{X}$ we have

$$
d \mathcal{F}_{\Gamma}\left[r_{0} ; \xi\right]=d F\left[\Gamma_{r_{0}} ; \xi \circ\left(\mathrm{I}+r_{0}\right)^{-1}\right]=d \mathcal{F}_{\Gamma_{r_{0}}}\left[0 ; \xi \circ\left(\mathrm{I}+r_{0}\right)^{-1}\right]
$$


Definition 3.4. (Higher order derivatives) Let $m \in \mathbb{N}$. We say that $f$ is $(m+1)$-times continuously (or $\mathscr{C}^{m+1}$ ) Gâteaux differentiable if it is $\mathscr{C}^{m}$ Gâteaux differentiable and

$$
U \ni r \mapsto d^{m} f\left[r ; \xi_{1}, \ldots, \xi_{m}\right]
$$

is continuously Gâteaux differentiable for all $m$-tuples $\left(\xi_{1}, \ldots, \xi_{m}\right) \in \mathcal{X}^{m}$. Then for all $r_{0} \in U$ the mapping

$$
\mathcal{X}^{m+1} \ni\left(\xi_{1}, \ldots, \xi_{m+1}\right) \mapsto d^{m+1} f\left[r_{0} ; \xi_{1}, \ldots, \xi_{m+1}\right] \in \mathcal{Y}
$$

is $(m+1)$-linear, symmetric and continuous. We say that $f$ is $\mathscr{C}^{\infty}$-Gâteaux differentiable if it is $\mathscr{C}^{m}$-Gâteaux differentiable for all $m \in \mathbb{N}$.

Proposition 3.5. Let $f: U \rightarrow \mathcal{Y}$ be $\mathscr{C}^{m}$-Gâteaux differentiable. Let us fix $r_{0} \in U$ and $\xi \in \mathcal{X}$. We set $\gamma(t)=f\left(r_{0}+t \xi\right)$.

i) The function of a real variable $\gamma$ is of class $\mathscr{C}^{m}$ in the neighborhood of zero and

$$
\gamma^{(m)}(t)=\left.\frac{d^{m}}{d t^{m}}\right|_{t=0} f\left(r_{0}+t \xi\right)=d^{m} f[r_{0} ; \underbrace{\xi, \ldots, \xi}_{m \text { times }}] .
$$

ii) We use the notation

$$
\frac{\partial^{m}}{\partial r^{m}} f\left[r_{0} ; \xi\right]=d^{m} f[r_{0} ; \underbrace{\xi, \ldots, \xi}_{m \text { times }}] .
$$

We then have

$d^{m} f\left[r_{0} ; \xi_{1}, \ldots, \xi_{m}\right]=\frac{1}{m !} \sum_{p=1}^{m}(-1)^{m-p} \sum_{1 \leq i_{1}<\cdots<i_{p} \leq m} \frac{\partial^{m}}{\partial r^{m}} f\left[r_{0} ; \xi_{i_{1}}+\ldots+\xi_{i_{p}}\right]$.

Thus the knowledge of $\frac{\partial^{m}}{\partial r^{m}} f\left[r_{0} ; \xi\right]$ suffices to determine the expression of $d^{m} f\left[r_{0} ; \xi_{1}, \ldots, \xi_{m}\right]$.

Proposition 3.6. Let $f: U \rightarrow \mathcal{Y}$ be $\mathscr{C}^{m}$-Gâteaux differentiable. Let us fix $r_{0} \in U$ and $\xi \in \mathcal{X}$ with $\xi$ sufficiently small. Then we have the following Taylor expansion with integral remainder :

$$
f\left(r_{0}+\xi\right)=\sum_{k=1}^{m-1} \frac{1}{k !} \frac{\partial^{k}}{\partial r^{k}} f\left[r_{0} ; \xi\right]+\int_{0}^{1} \frac{(1-\lambda)^{m}}{m !} \frac{\partial^{m}}{\partial r^{m}} f\left[r_{0}+\lambda \xi ; \xi\right] d \lambda .
$$

The chain and product rules are still available for $\mathscr{C}^{m}$-Gâteaux differentiable maps between Fréchet spaces. 


\section{Shape differentiability of boundary integral operators}

Let $x_{r}$ denote an element of $\Gamma_{r}$ and let $\boldsymbol{n}_{r}$ be the outer unit normal vector to $\Gamma_{r}$. When $r=0$ we write $\boldsymbol{n}_{0}=\boldsymbol{n}$. We denote by $d s\left(x_{r}\right)$ the area element on $\Gamma_{r}$.

In this section we want to establish the differentiability properties with respect to $r \in B^{\infty}(0, \varepsilon)$ of boundary integral operators $\mathcal{K}_{\Gamma_{r}}$ defined for a function $u_{r} \in H^{t}\left(\Gamma_{r}\right)$ by:

$$
\left(\mathcal{K}_{\Gamma_{r}} u_{r}\right)\left(x_{r}\right)=\int_{\Gamma_{r}} k_{r}\left(y_{r}, x_{r}-y_{r}\right) u_{r}\left(y_{r}\right) d s\left(y_{r}\right), x_{r} \in \Gamma_{r}
$$

and of potential operators $\mathcal{P}_{r}$ defined by:

$$
\left(\mathcal{P}_{r} u_{r}\right)(x)=\int_{\Gamma_{r}} k_{r}\left(y_{r}, x-y_{r}\right) u_{r}\left(y_{r}\right) d s\left(y_{r}\right), x \in \Omega_{r} \cup \Omega_{r}^{c},
$$

where $k_{r} \in \mathscr{C}^{\infty}\left(\Gamma_{r} \times\left(\mathbb{R}^{d} \backslash\{0\}\right)\right)$ is a pseudo-homogeneous kernel of class $-m$ with $m \in \mathbb{N}$.

We point out that we have to analyze mappings of the form $r \mapsto \mathcal{F}_{\Gamma}(r)$ where the domain of definition of $\mathcal{F}_{\Gamma}(r)$ varies with $r$. This is the main difficulty encountered in the calculus of shape variations. We propose different strategies according to the definition of the mapping $\mathcal{F}_{\Gamma}$.

(i) A first idea, quite classical (see [9, 21, 22]), is that instead of studying mappings $r \mapsto \mathcal{F}_{\Gamma}(r)$ where $\mathcal{F}_{\Gamma}(r)=u_{r}$ is a function defined on the boundary $\Gamma_{r}$, we consider the mapping

$$
r \mapsto u_{r} \circ(\mathrm{I}+r) .
$$

Typical examples of such functions $u_{r}$ are the normal vector $\boldsymbol{n}_{r}$ on $\Gamma_{r}$ and the kernel $k_{r}$ of a boundary integral operator $\mathcal{K}_{\Gamma_{r}}$ (see Examples 2.4 and 2.5]).

To formalize this, we define the transformation ("pullback") $\tau_{r}$ which maps a function $u_{r}$ defined on $\Gamma_{r}$ to the function $u_{r} \circ(\mathrm{I}+r)$ defined on $\Gamma$. For all $r \in B^{\infty}(0, \varepsilon)$, the transformation $\tau_{r}$ is linear and continuous from the function spaces $\mathscr{C}^{k}\left(\Gamma_{r}\right)$ and $H^{t}\left(\Gamma_{r}\right)$ to $\mathscr{C}^{k}(\Gamma)$ and $H^{t}(\Gamma)$, respectively, and admits an inverse. We have

$$
\left(\tau_{r} u_{r}\right)(x)=u_{r}(x+r(x)) \text { and }\left(\tau_{r}^{-1} u\right)\left(x_{r}\right)=u(x) .
$$

(ii) Next, for linear bounded operators between function spaces on the boundary, we use conjugation with the pullback $\tau_{r}$ : Instead of studying the mapping

$$
B^{\infty}(0, \varepsilon) \ni r \mapsto \mathcal{F}_{\Gamma}(r)=\mathcal{K}_{\Gamma_{r}} \in \mathscr{L}\left(H^{s}\left(\Gamma_{r}\right), H^{s+m}\left(\Gamma_{r}\right)\right)
$$

we consider the mapping

$$
B^{\infty}(0, \varepsilon) \ni r \mapsto \tau_{r} \mathcal{K}_{\Gamma_{r}} \tau_{r}^{-1} \in \mathscr{L}\left(H^{s}(\Gamma), H^{s+m}(\Gamma)\right) .
$$

We have for $u \in H^{s}(\Gamma)$ and $x \in \Gamma$ :

$$
\left(\tau_{r} \mathcal{K}_{\Gamma_{r}} \tau_{r}^{-1}\right)(u)(x)=\int_{\Gamma} k_{r}(y+r(y), x+r(x)-y-r(y)) u(y) J_{r}(y) d s(y),
$$


where $J_{r}$ is the Jacobian (the determinant of the Jacobian matrix) of the change of variables on the surface, mapping $x \in \Gamma$ to $x+r(x) \in \Gamma_{r}$.

(iii) The third case concerns potential operators acting from the boundary to the domain:

Each domain $\Omega$ is a countable union of compact subsets: $\Omega=\bigcup_{p \in \mathbb{N}} K_{p}$. For all $p \in \mathbb{N}$, there exists $\varepsilon_{p}>0$ such that $K_{p} \subset \bigcap_{r \in B\left(0, \varepsilon_{p}\right)} \Omega_{r}$. Thus, instead of studying the mapping

$$
B^{\infty}(0, \varepsilon) \ni r \mapsto \mathcal{F}_{\Gamma}(r)=\mathcal{P}_{r} \in \mathscr{L}\left(H^{s}\left(\Gamma_{r}\right), H^{s+m+\frac{1}{2}}\left(\Omega_{r}\right)\right)
$$

we can consider the mapping

$$
B^{\infty}\left(0, \varepsilon_{p}\right) \ni r \mapsto \mathcal{P}_{r} \tau_{r}^{-1} \in \mathscr{L}\left(H^{s}(\Gamma), H^{s+m+\frac{1}{2}}\left(K_{p}\right)\right) .
$$

We have for $u \in H^{s}(\Gamma)$

$$
\left(\mathcal{P}_{\Gamma}(r) \tau_{r}^{-1}\right)(u)(x)=\int_{\Gamma} k_{r}(y+r(y), x-y-r(y)) u(y) J_{r}(y) d s(y), \quad x \in K_{p} .
$$

Then passing to the limit $p \rightarrow \infty$ we can deduce the differentiability properties of the potentials on the whole domain $\Omega$. We use the analogous technique for the exterior domain $\Omega^{c}$.

In the framework of boundary integral equations, these approaches were introduced by Potthast [21, 22] in order to study the shape differentiability of solutions of acoustic boundary value problems.

Remark 4.1. In continuum mechanics, when the deformation $x \mapsto r(x)=$ $r_{0}(x)+t \xi(x)$ is interpreted as a flow with initial velocity field $\xi(x)$, one frequently considers two different derivatives of functions $u_{r}$ defined on $\Omega_{r}$. The material derivative $\dot{u}_{r}$ is computed by pulling $u_{r}$ back to the reference domain $\Omega$, thus by differentiating $r \mapsto \tau_{r} u_{r}=u_{r} \circ(\mathrm{I}+r)$. The shape derivative $u_{r}^{\prime}(x)$ at a point $x$ is defined by differentiating $u_{r}(x)$ directly. At $r=0$ the difference between the two derivatives is a convection term:

$$
\dot{u}_{0}=u_{0}^{\prime}+\xi \cdot \nabla u_{0} .
$$

This is easily seen from the definition of the material derivative

$$
\dot{u}_{r}(x)=d(\tau u)[0 ; \xi](x)=\left.\frac{d}{d t}\right|_{t=0} u_{t \xi}(x+t \xi(x))=d u[0 ; \xi](x)+\xi(x) \cdot \nabla u_{0}(x) \text {. }
$$

Relation (4.5) can be used to compute the shape derivative from the simpler material derivative, see [18, for an application.

In this terminology, the derivatives of boundary functions and operators in (i) and (ii) above would be analogous to material derivatives, whereas the derivatives of potentials in (iii) correspond to shape derivatives. Instead of formally defining the terms "material derivative" and "shape derivative", we prefer here to explain in each instance precisely which Gâteaux derivative 
is meant. We want to emphasize, however, that the shape derivatives of solutions of electromagnetic transmission problems can be obtained by using the three kinds of derivatives defined above. This will be explained in detail in Part II of this work. The construction is based on an integral representation of the solution of the transmission problem by potentials, the densities of which are solutions of boundary integral equations with operators of the type studied here. Thus the mapping from the given right hand side to the solution is a composition of boundary integral operators, inverses of boundary integral operators, and potential operators. By the chain rule, its derivative is then obtained by composing boundary integral operators, their inverses, and potential operators with derivatives of type (i), (ii), and (iii) above. The same structure gives the shape gradient of shape functionals that are defined from the solution of the transmission problem. In this case, also adjoints of the boundary integral operators have to be differentiated. This poses no new problem, because adjoints of operators with quasi-homogeneous kernels have quasi-homogeneous kernels, too.

\subsection{Gâteaux differentiability of coefficient functions}

For the analysis of the integral operators defined by (4.3) and (4.4), we first have to analyze coefficient functions such as the Jacobian of the change of variables $\Gamma \ni x \mapsto x+r(x) \in \Gamma_{r}$, or the normal vector $\boldsymbol{n}_{r}$ on $\Gamma_{r}$.

We use the standard surface differential operators as described in detail in $\left[20\right.$. For a vector function $\boldsymbol{v} \in \mathscr{C}^{k}\left(\mathbb{R}^{d}, \mathbb{C}^{d}\right)$ with $k \in \mathbb{N}^{*}$, we denote by $[\nabla \boldsymbol{v}]$ the matrix the $i$-th column of which is the gradient of the $i$-th component of $\boldsymbol{v}$, and we write $[\mathrm{D} \boldsymbol{v}]=[\nabla \boldsymbol{v}]^{\top}$. The tangential gradient of a scalar function $u \in \mathscr{C}^{k}(\Gamma, \mathbb{C})$ is defined by

$$
\nabla_{\Gamma} u=\nabla \tilde{u}_{\mid \Gamma}-\left(\nabla \tilde{u}_{\mid \Gamma} \cdot \boldsymbol{n}\right) \boldsymbol{n},
$$

where $\tilde{u}$ is an extension of $u$ to the whole space $\mathbb{R}^{d}$. For a vector function $\boldsymbol{u} \in \mathscr{C}^{k}\left(\Gamma, \mathbb{C}^{d}\right)$, we again denote by $\left[\nabla_{\Gamma} \boldsymbol{u}\right]$ the matrix the $i$-th column of which is the tangential gradient of the $i$-th component of $\boldsymbol{u}$ and we set $\left[\mathrm{D}_{\Gamma} \boldsymbol{u}\right]=$ $\left[\nabla_{\Gamma} \boldsymbol{u}\right]^{\top}$.

We define the surface divergence of a vector function $\boldsymbol{u} \in \mathscr{C}^{k}\left(\Gamma, \mathbb{C}^{d}\right)$ by

$$
\operatorname{div}_{\Gamma} \boldsymbol{u}=\operatorname{div} \tilde{\boldsymbol{u}}_{\mid \Gamma}-\left(\left[\nabla \tilde{\boldsymbol{u}}_{\mid \Gamma}\right] \boldsymbol{n} \cdot \boldsymbol{n}\right)=\operatorname{div} \tilde{\boldsymbol{u}}_{\mid \Gamma}-\left(\boldsymbol{n} \cdot \frac{\partial \boldsymbol{u}}{\partial \boldsymbol{n}}\right),
$$

where $\tilde{\boldsymbol{u}}$ is an extension of $\boldsymbol{u}$ to the whole space $\mathbb{R}^{d}$. These definitions do not depend on the choice of the extension.

The surface Jacobian $J_{r}$ is given by the formula $J_{r}=\mathrm{Jac}_{\Gamma}(\mathrm{I}+r)=\left\|w_{r}\right\|$ with

$$
w_{r}=\operatorname{cof}\left(\mathrm{I}+\mathrm{D} r_{\mid \Gamma}\right) \boldsymbol{n}=\operatorname{det}\left(\mathrm{I}+\mathrm{D} r_{\mid \Gamma}\right)\left(\mathrm{I}+\mathrm{D} r_{\mid \Gamma}\right)^{-1^{\top}} \boldsymbol{n},
$$

where $\operatorname{cof}(A)$ means the matrix of cofactors of the matrix $A$, and the normal vector $\boldsymbol{n}_{r}$ is given by

$$
\boldsymbol{n}_{r}=\tau_{r}^{-1}\left(\frac{w_{r}}{\left\|w_{r}\right\|}\right)
$$


The first derivative at $r=0$ of these functions are well known, we refer for instance to Henrot-Pierre [9]. Here we present a method that allows to obtain higher order derivatives.

Lemma 4.2. The functional $\mathcal{J}$ mapping $r \in B^{\infty}(0, \varepsilon)$ to the Jacobian $J_{r} \in$ $\mathscr{C}^{\infty}(\Gamma, \mathbb{R})$ is $\mathscr{C}^{\infty}$-Gâteaux differentiable and its first derivative at $r_{0}$ is given for $\xi \in \mathscr{C}^{\infty}\left(\Gamma, \mathbb{R}^{d}\right)$ by

$$
d \mathcal{J}\left[r_{0}, \xi\right]=J_{r_{0}}\left(\tau_{r_{0}} \operatorname{div}_{\Gamma_{r_{0}}}\left(\tau_{r_{0}}^{-1} \xi\right)\right) .
$$

Proof. We just have to prove the $\mathscr{C}^{\infty}$-Gâteaux differentiability of

$$
\mathcal{W}: B^{\infty}(0, \varepsilon) \ni r \mapsto w_{r}=\operatorname{cof}\left(\mathrm{I}+\mathrm{D} r_{\mid \Gamma}\right) \boldsymbol{n} \in \mathscr{C}^{\infty}(\Gamma) .
$$

We use a local coordinate system. Assume that $\Gamma$ is parametrized by an atlas $\left(\mathcal{O}_{i}, \phi_{i}\right)_{1 \leq i \leq p}$ then $\Gamma_{r}$ can be parametrized by the atlas $\left(\mathcal{O}_{i},(\mathrm{I}+r) \circ \phi_{i}\right)_{1 \leq i \leq p}$. For any $x \in \Gamma$, let us denote by $e_{1}(x), e_{2}(x), \ldots, e_{d-1}(x)$ a vector basis of the tangent plane to $\Gamma$ at $x$. A basis of the tangent plane to $\Gamma_{r}$ at $x+r(x)$ is then given by

$$
e_{i}(r, x)=[(\mathrm{I}+\mathrm{D} r)(x)] e_{i}(x) \quad \text { for } i=1, \ldots, d-1 .
$$

Notice that for $i=1, \ldots, d-1$ the mapping $B^{\infty}(0, \varepsilon) \ni r \mapsto e_{i}(r) \in$ $\mathscr{C}^{\infty}\left(\Gamma, \mathbb{R}^{d}\right)$ is $\mathscr{C}^{\infty}$-Gâteaux differentiable. Its first derivative is $d e_{i}\left[r_{0} ; \xi\right]=$ $[\mathrm{D} \xi] e_{i}\left(r_{0}\right)$, and higher order derivatives vanish. We have

$$
w_{r}(x)=\frac{\bigwedge_{i=1}^{d-1} e_{i}(r, x)}{\left|\bigwedge_{i=1}^{d-1} e_{i}(x)\right|}
$$

where the wedge means the exterior product. Since the mappings $r \mapsto e_{i}(r)$, for $i=1, \ldots, d-1$ are $\mathscr{C}^{\infty}$-Gâteaux differentiable, by composition the mapping $W$ is, too. We compute now the derivatives using formulas (3.2)-(3.3). Let $\xi \in \mathscr{C}^{\infty}\left(\Gamma, \mathbb{R}^{d}\right)$ and $t$ small enough. We have at $r_{0} \in B^{\infty}(0, \varepsilon)$

$$
\frac{\partial^{m} \mathcal{W}}{\partial r^{m}}\left[r_{0}, \xi\right]=\left.\frac{\partial^{m}}{\partial t^{m}}\right|_{t=0} \frac{\bigwedge_{i=1}^{d-1}\left(\mathrm{I}+D r_{0}+t D \xi\right) e_{i}(x)}{\left|\bigwedge_{i=1}^{d-1} e_{i}(x)\right|} .
$$

To simplify this expression one notes that

$$
\begin{aligned}
{[\mathrm{D} \xi(x)] e_{i}(x) } & =[\mathrm{D} \xi(x)]\left[\left(\mathrm{I}+\mathrm{D} r_{0}\right)(x)\right]^{-1}\left[\left(\mathrm{I}+\mathrm{D} r_{0}\right)(x)\right] e_{i}(x) \\
& =[\mathrm{D} \xi(x)]\left[\mathrm{D}\left(\mathrm{I}+r_{0}\right)^{-1}\left(x+r_{0}(x)\right)\right]\left[\left(\mathrm{I}+\mathrm{D} r_{0}\right)(x)\right] e_{i}(x) \\
& =\left[\tau_{r_{0}} \mathrm{D}\left(\tau_{r_{0}}^{-1} \xi\right)(x)\right] e_{i}\left(r_{0}, x\right)=\left[\tau_{r_{0}} \mathrm{D}_{\Gamma_{r_{0}}}\left(\tau_{r_{0}}^{-1} \xi\right)(x)\right] e_{i}\left(r_{0}, x\right) .
\end{aligned}
$$

Now given a $(d \times d)$ matrix $A$ we have

$$
\sum_{i=1}^{d-1} \cdots \wedge e_{i-1} \times A e_{i} \wedge e_{i+1} \wedge \cdots=\left(\operatorname{Trace}(A) \mathrm{I}-A^{\top}\right) \bigwedge_{i=1}^{d-1} e_{i}
$$


Thus we have with $A=\left[\tau_{r_{0}} \mathrm{D}_{\Gamma_{r_{0}}}\left(\tau_{r_{0}}^{-1} \xi\right)\right]$ and $B_{0}=\mathrm{I}, B_{1}(A)=\operatorname{Trace}(A) \mathrm{I}-A^{\top}$

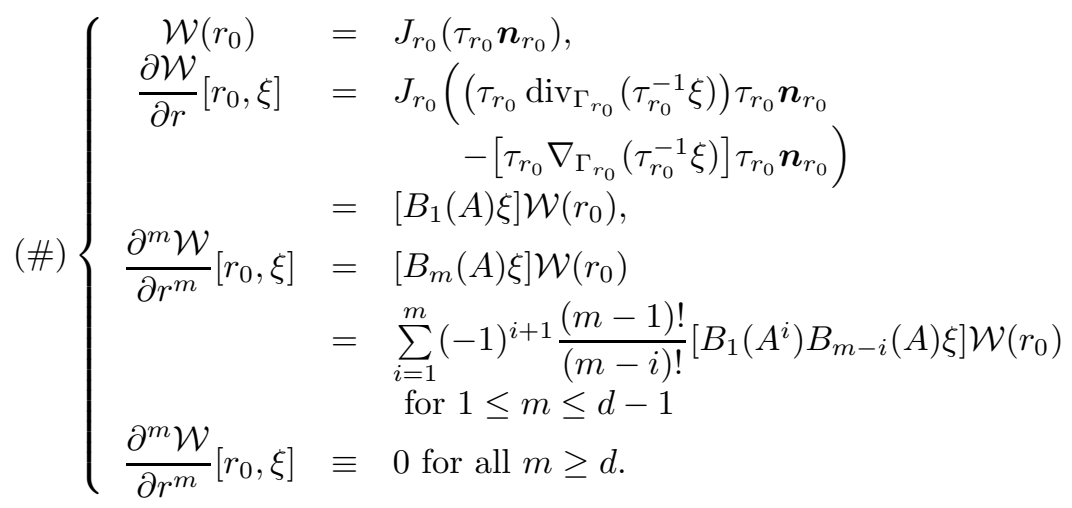

It follows that

$$
\begin{aligned}
\frac{\partial \mathcal{J}}{\partial r}\left[r_{0}, \xi\right] & =\frac{1}{\left\|\mathcal{W}\left(r_{0}\right)\right\|} \frac{\partial \mathcal{W}}{\partial r}\left[r_{0}, \xi\right] \cdot \mathcal{W}\left(r_{0}\right) \\
& =\frac{\partial \mathcal{W}}{\partial r}\left[r_{0}, \xi\right] \cdot \tau_{r_{0}} \boldsymbol{n}_{r_{0}}=J_{r_{0}}\left(\tau_{r_{0}} \operatorname{div}_{\Gamma_{r_{0}}}\left(\tau_{r_{0}}^{-1} \xi\right)\right)
\end{aligned}
$$

From (\#) we deduce easily the Gâteaux differentiability of $r \mapsto \tau_{r} \boldsymbol{n}_{r}$.

Lemma 4.3. The mapping $\mathcal{N}$ from $r \in B^{\infty}(0, \varepsilon)$ to $\tau_{r} \boldsymbol{n}_{r}=\boldsymbol{n}_{r} \circ(\mathrm{I}+r) \in$ $\mathscr{C}^{\infty}\left(\Gamma, \mathbb{R}^{d}\right)$ is $\mathscr{C}^{\infty}$-Gâteaux-differentiable and its first derivative at $r_{0}$ is defined for $\xi \in \mathscr{C}^{\infty}\left(\Gamma, \mathbb{R}^{d}\right)$ by:

$$
\frac{\partial \mathcal{N}}{\partial r}\left[r_{0}, \xi\right]=-\left[\tau_{r_{0}} \nabla_{\Gamma_{r_{0}}}\left(\tau_{r_{0}}^{-1} \xi\right)\right] \mathcal{N}\left(r_{0}\right) .
$$

Proof. Using the preceding proof, we find

$$
\begin{aligned}
\frac{\partial \mathcal{N}}{\partial r}\left[r_{0}, \xi\right] & =\frac{1}{\left\|\mathcal{W}\left(r_{0}\right)\right\|} \frac{\partial \mathcal{W}}{\partial r}\left[r_{0}, \xi\right]-\frac{1}{\left\|\mathcal{W}\left(r_{0}\right)\right\|^{3}}\left(\frac{\partial \mathcal{W}}{\partial r}\left[r_{0}, \xi\right] \cdot \mathcal{W}\left(r_{0}\right)\right) \mathcal{W}\left(r_{0}\right) \\
& =J_{r_{0}}^{-1}\left(\frac{\partial \mathcal{W}}{\partial r}\left[r_{0}, \xi\right]-\left(\frac{\partial \mathcal{W}}{\partial r}\left[r_{0}, \xi\right] \cdot\left(\tau_{r_{0}} \boldsymbol{n}_{r_{0}}\right)\right)\right) \tau_{r_{0}} \boldsymbol{n}_{r_{0}} \\
& =-\left[\tau_{r_{0}} \nabla_{\Gamma_{r_{0}}}\left(\tau_{r_{0}}^{-1} \xi\right)\right] \tau_{r_{0}} \boldsymbol{n}_{r_{0}} .
\end{aligned}
$$

To obtain higher order shape derivatives of these mappings one can use the equalities (\#) and

$$
(*)\left\{\begin{array}{c}
\left\|\tau_{r} \boldsymbol{n}_{r}\right\| \\
\frac{\partial^{m} \mathcal{N} \cdot \mathcal{N}}{\partial r^{m}}\left[r_{0}, \xi\right] \equiv 0 \text { for all } m \geq 1 .
\end{array}\right.
$$

For example, we have at $r=0$ in the direction $\xi \in \mathscr{C}^{\infty}\left(\Gamma, \mathbb{R}^{d}\right)$ :

$$
\frac{\partial \mathcal{J}}{\partial r}[0, \xi]=\operatorname{div}_{\Gamma} \xi \text { and } \frac{\partial \mathcal{N}}{\partial r}[0, \xi]=-\left[\nabla_{\Gamma} \xi\right] \boldsymbol{n}
$$


Using Proposition 3.5, we obtain

$\frac{\partial^{2} \mathcal{J}}{\partial r^{2}}\left[0, \xi_{1}, \xi_{2}\right]=-\operatorname{Trace}\left(\left[\nabla_{\Gamma} \xi_{2}\right]\left[\nabla_{\Gamma} \xi_{1}\right]\right)+\operatorname{div}_{\Gamma} \xi_{1} \cdot \operatorname{div}_{\Gamma} \xi_{2}+\left(\left[\nabla_{\Gamma} \xi_{1}\right] \boldsymbol{n} \cdot\left[\nabla_{\Gamma} \xi_{2}\right] \boldsymbol{n}\right)$.

Notice that $\operatorname{Trace}\left(\left[\nabla_{\Gamma} \xi_{2}\right]\left[\nabla_{\Gamma} \xi_{1}\right]\right)=\operatorname{Trace}\left(\left[\nabla_{\Gamma} \xi_{1}\right]\left[\nabla_{\Gamma} \xi_{2}\right]\right)$.

$$
\frac{\partial^{2} \mathcal{N}}{\partial r^{2}}\left[0, \xi_{1}, \xi_{2}\right]=\left[\nabla_{\Gamma} \xi_{2}\right]\left[\nabla_{\Gamma} \xi_{1}\right] \boldsymbol{n}+\left[\nabla_{\Gamma} \xi_{1}\right]\left[\nabla_{\Gamma} \xi_{2}\right] \boldsymbol{n}-\left(\left[\nabla_{\Gamma} \xi_{1}\right] \boldsymbol{n} \cdot\left[\nabla_{\Gamma} \xi_{2}\right] \boldsymbol{n}\right) \boldsymbol{n}
$$

In the last section we give a second method to obtain higher order derivatives using the Gâteaux derivatives of the surface differential operators.

Remark 4.4. The computation of the derivatives does not require more than the first derivative of the deformations $\xi$. As a consequence for hypersurfaces of class $\mathscr{C}^{k+1}$, it suffices to consider deformations of class $\mathscr{C}^{k+1}$ to conserve the regularity $\mathscr{C}^{k}$ of the Jacobian and of the normal vector by differentiation.

\subsection{Gâteaux differentiability of pseudo-homogeneous kernels}

The following theorem establishes sufficient conditions for the Gâteaux differentiability of the boundary integral operators described above.

Theorem 4.5. Let $p \in \mathbb{N}$. We set $(\Gamma \times \Gamma)^{*}=\{(x, y) \in \Gamma \times \Gamma ; x \neq y\}$. Assume that the following two conditions are satisfied:

1) For all fixed $(x, y) \in(\Gamma \times \Gamma)^{*}$ the function

$$
\begin{aligned}
f: B^{\infty}(0, \varepsilon) & \rightarrow \mathbb{C} \\
r & \mapsto k_{r}(y+r(y), x+r(x)-y-r(y)) J_{r}(y)
\end{aligned}
$$

is $\mathscr{C}^{p+1}$ - Gâteaux differentiable.

2) The functions $(y, x-y) \mapsto f\left(r_{0}\right)(y, x-y)$ and

$$
(y, x-y) \mapsto d^{l} f\left[r_{0}, \xi_{1}, \ldots, \xi_{l}\right](y, x-y)
$$

are pseudo-homogeneous of class $-m$ for all $r_{0} \in B^{\infty}(0, \varepsilon)$, for all $l=$ $1, \ldots, p+1$ and for all $\xi_{1}, \ldots, \xi_{p+1} \in \mathscr{C}^{\infty}\left(\Gamma, \mathbb{R}^{d}\right)$.

Then for any $s \in \mathbb{R}$ the mapping

$$
\begin{aligned}
B^{\infty}(0, \varepsilon) & \rightarrow \mathscr{L}\left(H^{s}(\Gamma), H^{s+m}(\Gamma)\right) \\
r & \mapsto \tau_{r} \mathcal{K}_{\Gamma_{r}} \tau_{r}^{-1}
\end{aligned}
$$

is $\mathscr{C}^{p}$-Gâteaux differentiable and

$$
d^{p}\left(\tau_{r} \mathcal{K}_{\Gamma_{r}} \tau_{r}^{-1}\right)\left[r_{0}, \xi_{1}, \ldots, \xi_{p}\right] u(x)=\int_{\Gamma} d^{p} f\left[r_{0}, \xi_{1}, \ldots, \xi_{p}\right](y, x-y) u(y) d s(y)
$$

Proof. We use the linearity of the integral and Taylor expansion with integral remainder. We do the proof for $p=1$ only. Let $r_{0} \in B^{\infty}(0, \varepsilon), \xi \in \mathscr{C}^{\infty}\left(\Gamma, \mathbb{R}^{d}\right)$ and $t$ small enough such that $r_{0}+t \xi \in B^{\infty}(0, \varepsilon)$. We have

$$
\begin{aligned}
f\left(r_{0}+t \xi, x, y\right)-f\left(r_{0}, y, x-y\right) & =t \frac{\partial f}{\partial r}\left[r_{0}, \xi\right](y, x-y) \\
& +t^{2} \int_{0}^{1}(1-\lambda) \frac{\partial^{2} f}{\partial r^{2}}\left[r_{0}+\lambda t \xi, \xi\right](y, x-y) d \lambda .
\end{aligned}
$$

We have to verify that each term in this equality is a kernel of an operator mapping $H^{s}(\Gamma)$ to $H^{s+m}(\Gamma)$. The two first terms in the left hand side are 
pseudo-homogeneous kernels of class $-m$ and by hypothesis $\frac{\partial f}{\partial r}\left[r_{0}, \xi\right]$ is also a kernel of class $-m$. It remains to prove that the operator with kernel

$$
(x, y) \mapsto \int_{0}^{1}(1-\lambda) \frac{\partial^{2} f}{\partial r^{2}}\left[r_{0}+\lambda t \xi, \xi\right](x, y) d \lambda
$$

acts from $H^{s}(\Gamma)$ to $H^{s+m}(\Gamma)$ with norm bounded uniformly in $t$. Since $\frac{\partial^{2} f}{\partial r^{2}}\left[r_{0}+\lambda t \xi, \xi\right]$ is pseudo-homogeneous of class $-m$ for all $\lambda \in[0,1]$, it suffices to use Lebesgue's theorem in order to invert the integration with respect to the variable $\lambda$ and the integration with respect to $y$ on $\Gamma$.

$$
\begin{aligned}
& \left\|\int_{\Gamma}\left(\int_{0}^{1}(1-\lambda) \frac{\partial^{2} f}{\partial r^{2}}\left[r_{0}+\lambda t \xi, \xi\right](x, y) d \lambda\right) u(y) d s(y)\right\|_{H^{s+m}(\Gamma)} \\
& =\left\|\int_{0}^{1}(1-\lambda)\left(\int_{\Gamma} \frac{\partial^{2} f}{\partial r^{2}}\left[r_{0}+\lambda t \xi, \xi\right](x, y) u(y) d s(y)\right) d \lambda\right\|_{H^{s+m}(\Gamma)} \\
& \leq \sup _{\lambda \in[0,1]}\left\|\left(\int_{\Gamma} \frac{\partial^{2} f}{\partial r^{2}}\left[r_{0}+\lambda t \xi, \xi\right](x, y) u(y) d s(y)\right)\right\|_{H^{s+m}(\Gamma)} \\
& \leq C\|u\|_{H^{s}(\Gamma)} .
\end{aligned}
$$

We then have

$$
\begin{aligned}
\frac{1}{t}\left(\int _ { \Gamma } f \left(r_{0}\right.\right. & \left.+t \xi, x, y) u(y) d s(y)-\int_{\Gamma} f\left(r_{0}, x, y\right) u(y) d s(y)\right) \\
& =\int_{\Gamma} \frac{\partial f}{\partial r}\left[r_{0}, \xi\right](x, y) u(y) d s(y) \\
& +t \int_{\Gamma}\left(\int_{0}^{1}(1-\lambda) \frac{\partial^{2} f}{\partial r^{2}}\left[r_{0}+\lambda t \xi, \xi\right](x, y) d \lambda\right) u(y) d s(y)
\end{aligned}
$$

We pass to the operator norm limit $t \rightarrow 0$ and we obtain the first Gâteaux derivative. For higher order derivatives it suffices to write the proof with $d^{p} f\left[r_{0}, \xi_{1}, \ldots, \xi_{k}\right]$ instead of $f$. The linearity, the symmetry and the continuity of the first derivative are deduced from the corresponding properties of the derivatives of the kernel.

Now we will consider some particular classes of pseudo-homogeneous kernels.

Corollary 4.6. Assume that the kernels $k_{r}$ are of the form

$$
k_{r}\left(y_{r}, x_{r}-y_{r}\right)=G\left(x_{r}-y_{r}\right)
$$

where $G \in \mathscr{C}^{\infty}\left(\mathbb{R}^{d} \backslash\{0\}\right)$ is a pseudo-homogeneous kernel of class $-m, m \in \mathbb{N}$, which does not depend on $r$. Then the mapping

$$
\begin{aligned}
B^{\infty}(0, \varepsilon) & \rightarrow \mathscr{L}\left(H^{t}(\Gamma), H^{t+m}(\Gamma)\right) \\
r & \mapsto \tau_{r} \mathcal{K}_{\Gamma_{r}} \tau_{r}^{-1}
\end{aligned}
$$

is $\mathscr{C}^{\infty}$-Gâteaux differentiable and the kernel of the first derivative at $r=0$ is defined for $\xi \in \mathscr{C}^{\infty}\left(\Gamma, \mathbb{R}^{d}\right)$ by

$$
d f[0, \xi]=(\xi(x)-\xi(y)) \cdot \nabla G(x-y)+G(x-y) \operatorname{div}_{\Gamma} \xi(y) .
$$


Proof. For fixed $(x, y) \in(\Gamma \times \Gamma)^{*}$, consider the mapping

$$
f: B^{\infty}(0, \varepsilon) \ni r \mapsto f(r, x, y)=G(x+r(x)-y-r(y)) J_{r}(y) \in \mathbb{C} .
$$

By Theorem 4.5 we have to prove that $r \mapsto f(r)$ is $\mathscr{C}^{\infty}$-Gâteaux differentiable and that each derivative defines a pseudo-homogeneous kernel of class $-m$. $\triangleright$ Step 1:

First we prove that for fixed $(x, y) \in(\Gamma \times \Gamma)^{*}$ the mapping $r \mapsto f(r, x, y)$ is infinitely Gâteaux differentiable on $B^{\infty}(0, \varepsilon)$. By Lemma 4.2 the mapping $r \mapsto J_{r}(y)$ is infinitely Gâteaux differentiable on $B^{\infty}(0, \varepsilon)$, the mapping $r \mapsto$ $x+r(x)$ is also infinitely Gâteaux differentiable on $B^{\infty}(0, \varepsilon)$ and the kernel $G$ is of class $\mathscr{C}^{\infty}$ on $\mathbb{R}^{d} \backslash\{0\}$. Being composed of infinitely Gâteaux differentiable maps, the mapping $r \mapsto f(r, x, y)$ is, too.

$\triangleright$ Step 2:

We then prove that each derivative defines a pseudo-homogeneous kernel of class $-m$, that is to say that for all $p \in \mathbb{N}$ and for any $p$-tuple $\left(\xi_{1}, \ldots, \xi_{p}\right)$ the function

$$
(x, y) \mapsto d^{p} f\left[r_{0}, \xi_{1}, \ldots, \xi_{p}\right](x, y)
$$

is pseudo-homogeneous of class $-m$. By formula (3.3), it remains to write the proof for the function $\frac{\partial^{p}}{\partial r^{p}} f\left[r_{0}, \xi\right]$ with $\xi \in \mathscr{C}^{\infty}\left(\Gamma, \mathbb{R}^{d}\right)$. The Leibniz formula gives

$\frac{\partial^{p}}{\partial r^{p}} f\left[r_{0}, \xi\right](x, y)=\sum_{l=0}^{p}\left(\begin{array}{c}p \\ l\end{array}\right) \frac{\partial^{l}}{\partial r^{l}}\{G(x+r(x)-y-r(y))\}\left[r_{0}, \xi\right] \frac{\partial^{p-l} \mathcal{J}}{\partial r^{p-l}}\left[r_{0}, \xi\right](y)$.

Since $\frac{\partial^{p-l} \mathcal{J}}{\partial r^{p-l}}\left[r_{0}, \xi\right] \in \mathscr{C}^{\infty}(\Gamma, \mathbb{R})$, we have to prove that

$$
(x, y) \mapsto \frac{\partial^{l}}{\partial r^{l}}\{G(x+r(x)-y-r(y))\}\left[r_{0}, \xi\right]
$$

defines a pseudo-homogeneous kernel of class $-m$. We have

$$
\begin{aligned}
& \frac{\partial^{l}}{\partial r^{l}}\{G(x+r(x)-y-r(y))\}\left[r_{0} ; \xi\right] \\
& \quad=\mathrm{D}^{l} G\left[x+r_{0}(x)-y-r_{0}(y) ; \xi(x)-\xi(y), \ldots, \xi(x)-\xi(y)\right] .
\end{aligned}
$$

By definition, $G(z)$ admits the following asymptotic expansion when $z$ tends to zero:

$$
G(z)=G_{m}(z)+\sum_{j=1}^{N-1} G_{m+j}(z)+G_{m+N}(z)
$$

where $G_{m+j}$ is homogeneous of class $-(m+j)$ for $j=0, \ldots, N-1$ and $G_{m+N}$ is of arbitrary regularity. Using Taylor expansion, the following result is easy to see:

Lemma 4.7. Let the kernel $G_{m}(z)$ be homogeneous of class $-m$ and $\xi \in$ $\mathscr{C}^{\infty}\left(\Gamma, \mathbb{R}^{d}\right)$. Then the function

$$
(x, y-x) \mapsto D^{l} G_{m}\left[x+r_{0}(x)-y-r_{0}(y) ; \xi(x)-\xi(y), \ldots, \xi(x)-\xi(y)\right]
$$


is pseudo-homogeneous of class $-m$.

By taking derivatives in the expansion (4.8) we conclude that $\frac{\partial^{l}}{\partial r^{l}}\{G(x+r(x)-y-r(y))\}\left[r_{0} ; \xi\right]$ is pseudo-homogeneous of class $-m$ too. This ends the proof of the corollary.

Theorem 4.8. Let $s \in \mathbb{R}$. Let $G(z)$ be a pseudo-homogeneous kernel of class $-(m+1)$ with $m \in \mathbb{N}$. Let us fix a compact subdomain $K_{p}$ of $\Omega$. Assume that for all $r \in B^{\infty}\left(0, \varepsilon_{p}\right)$, we have $k_{r}\left(y_{r}, x-y_{r}\right)=G\left(x-y_{r}\right)$. Then the mapping

$$
\begin{aligned}
B_{\varepsilon}^{\infty} & \rightarrow \mathscr{L}\left(H^{s-\frac{1}{2}}(\Gamma), H^{s+m}\left(K_{p}\right)\right) \\
r & \mapsto \mathcal{P}_{r} \tau_{r}^{-1}
\end{aligned}
$$

is infinitely Gâteaux differentiable and

$$
\begin{aligned}
d^{p}\left(\mathcal{P}_{r} \tau_{r}^{-1}\right)\left[r_{0}, \xi_{1}\right. & \left.\ldots, \xi_{p}\right] u(x) \\
& =\int_{\Gamma} d^{p}\left\{G(x-y-r(y)) J_{r}(y)\right\}\left[r_{0}, \xi_{1}, \ldots, \xi_{p}\right] u(y) d s(y) .
\end{aligned}
$$

Its first derivative at $r=0$ in the direction $\xi \in \mathscr{C}^{\infty}\left(\Gamma, \mathbb{R}^{d}\right)$ is the integral operator denoted by $\mathcal{P}^{(1)}$ with kernel

$$
-\xi(y) \cdot \nabla^{z} G(x-y)+G(x-y) \operatorname{div}_{\Gamma} \xi(y) .
$$

The operator $\mathcal{P}^{(1)}$ can be extended to a continuous linear operator from $H^{s-\frac{1}{2}}(\Gamma)$ to $H^{s+m}(\Omega)$ and $H_{l o c}^{s+m}\left(\overline{\Omega^{c}}\right)$.

Proof. The kernel and its higher order derivatives are of class $\mathscr{C}^{\infty}$ on $K_{p}$. Writing $\Omega$ as an increasing union of compact subsets, we can define a shape derivative on the whole domain $\Omega$. Let us look at the first derivative: The term $G(x-y) \operatorname{div}_{\Gamma} \xi(y)$ has the same regularity as $G(x-y)$ when $x-y$ tends to zero wheareas $\xi(y) \cdot \nabla G(x-y)$ loses one order of regularity. As a consequence, since the kernel is of class $-(m+1)$, its first derivative acts from $H^{s-\frac{1}{2}}(\Gamma)$ to $H^{s+m}(\Omega)$ and $H_{l o c}^{s+m}\left(\overline{\Omega^{c}}\right)$.

Remark 4.9. We conclude that the boundary integral operators are smooth with respect to the domain whereas the potential operators lose one order of regularity at each derivation. We point out that we do not need more than the first derivative of the deformations $\xi$ to compute the Gâteaux derivatives of any order of these integral operators.

Example 4.10. (Acoustic single layer potential) Let $d=2$ or $d=3$ and $s \in \mathbb{R}$. We denote by $\Psi_{\kappa}^{r}$ the single layer potential defined for $u_{r} \in H^{s}\left(\Gamma_{r}\right)$ with the fundamental solution $G_{a}$ of the Helmholtz equation (see Example 2.4)

$$
\Psi_{\kappa}^{r} u_{r}(x)=\int_{\Gamma_{r}} G_{a}\left(\kappa, x-y_{r}\right) u_{r}\left(y_{r}\right) d s\left(y_{r}\right), x \in \mathbb{R}^{d} \backslash \Gamma_{r} .
$$

Let $V_{\kappa}^{r}$ its trace on $\Gamma_{r}$

$$
V_{\kappa}^{r} u_{r}(x)=\int_{\Gamma_{r}} G_{a}\left(\kappa, x-y_{r}\right) u_{r}\left(y_{r}\right) d s\left(y_{r}\right), x \in \Gamma_{r} .
$$


Since $G_{a}$ is pseudo-homogeneous of class -1 , the mapping

$$
\begin{aligned}
B^{\infty}(0, \varepsilon) & \rightarrow \mathscr{L}\left(H^{s}(\Gamma), H^{s+1}(\Gamma)\right) \\
r & \mapsto \tau_{r} V_{\kappa}^{r} \tau_{r}^{-1}
\end{aligned}
$$

is infinitely Gâteaux differentiable. The mapping

$$
\begin{array}{cl}
B^{\infty}\left(0, \varepsilon_{p}\right) & \rightarrow \mathscr{L}\left(H^{s}(\Gamma), H^{s+\frac{1}{2}}\left(K_{p}\right)\right) \\
r & \mapsto \tau_{r} \Psi_{\kappa}^{r} \tau_{r}^{-1}
\end{array}
$$

is infinitely differentiable and its first derivative at $r=0$ can be extended to a linear continuous operator from $H^{s}(\Gamma)$ to $H^{s+\frac{1}{2}}(\Omega) \cup H_{l o c}^{s+\frac{1}{2}}\left(\overline{\Omega^{c}}\right)$.

Similar results can be deduced for the elastic single layer potential.

Example 4.11. (Acoustic double layer kernel) Let $d=2$ or $d=3$ and $t \in \mathbb{R}$. We denote by $D_{\kappa}^{r}$ the boundary integral operator defined for $u_{r} \in H^{t}\left(\Gamma_{r}\right)$ by

$$
D_{\kappa}^{r} u_{r}(x)=\int_{\Gamma_{r}} \boldsymbol{n}_{r}\left(x_{r}\right) \cdot \nabla G_{a}\left(\kappa, y_{r}-x_{r}\right) u_{r}\left(y_{r}\right) d s\left(y_{r}\right) .
$$

The mapping

$$
\begin{aligned}
& B^{\infty}(0, \varepsilon) \rightarrow \mathscr{L}\left(H^{t}(\Gamma), H^{t+1}(\Gamma)\right) \\
& r \quad \mapsto \tau_{r} D_{\kappa}^{r} \tau_{r}^{-1}
\end{aligned}
$$

is $\mathscr{C}^{\infty}$-Gâteaux differentiable .

Indeed the mapping

$$
B^{\infty}(0, \varepsilon) \ni r \mapsto g(r, x, y)=\left(\tau_{r} \boldsymbol{n}_{r}\right)(x) \cdot(x+r(x)-y-r(y))
$$

is $\mathscr{C}^{\infty}$ Gâteaux differentiable and by using a local coordinate system (see [22]) we prove (when $d=3$ ) that the Gâteaux derivatives behaves as $|x-y|^{2}$ when $x-y \rightarrow 0$. We use the same notations as in the proof of Lemma 4.2, Fix $x \in \Gamma$ and set $g_{x}(r, y)=g(r, x, y)$. We have that $g_{x} \in \mathscr{C}^{\infty}\left(B^{\infty}(0, \varepsilon) \times \Gamma, \mathbb{R}\right)$. If $\Gamma$ is parametrised by the atlas $\left(\mathcal{O}_{i}, \phi_{i}\right)_{1 \leq i \leq p}$ then when $x \in \Gamma_{i}=\phi_{i}\left(\mathcal{O}_{i}\right) \cap \Gamma$ we can write $x=\phi_{i}\left(\eta_{1}^{x}, \eta_{2}^{x}\right)$ where $\left(\eta_{1}^{x}, \eta_{2}^{x}\right) \in \mathcal{O}_{i}$. The tangent plane to $\Gamma$ at $x$ is generated by the vectors $e_{1}(x)=\frac{\partial \phi_{i}}{\partial \eta_{1}}\left(\eta_{1}^{x}, \eta_{2}^{x}\right)$ and $e_{2}(x)=\frac{\partial \phi_{i}}{\partial \eta_{2}}\left(\eta_{1}^{x}, \eta_{2}^{x}\right)$. Thus $g_{x}\left(r, \phi_{i}\left(\eta_{1}, \eta_{2}\right)\right)$ has the expression

$$
\begin{aligned}
& \frac{(\mathrm{I}+\mathrm{D} r) \frac{\partial \phi_{i}}{\partial \eta_{1}}\left(\eta_{1}^{x}, \eta_{2}^{x}\right) \wedge(\mathrm{I}+D r) \frac{\partial \phi_{i}}{\partial \eta_{2}}\left(\eta_{1}^{x}, \eta_{2}^{x}\right)}{\left|(\mathrm{I}+D r) \frac{\partial \phi_{i}}{\partial \eta_{1}}\left(\eta_{1}^{x}, \eta_{2}^{x}\right) \wedge(\mathrm{I}+D r) \frac{\partial \phi_{i}}{\partial \eta_{2}}\left(\eta_{1}^{x}, \eta_{2}^{x}\right)\right|} \\
& \cdot\left((\mathrm{I}+r) \circ \phi_{i}\left(\eta_{1}^{x}, \eta_{2}^{x}\right)-(\mathrm{I}+r) \circ \phi_{i}\left(\eta_{1}, \eta_{2}\right)\right)
\end{aligned}
$$

Using Taylor expansion we have when $y \rightarrow x$

$$
g_{x}(r, y)=0+\mathrm{D} g_{x}(r)[x ; y-x]+\frac{1}{2} \mathrm{D}^{2} g_{x}(r)[x ; y-x, y-x]+\ldots
$$

Writing $g_{x}(r)=\left(g_{x}(r) \circ \phi_{i}\right) \circ \phi_{i}^{-1}$, we have for all $r \in B^{\infty}(0, \epsilon)$ that

$$
\mathrm{D} g_{x}(r)=\mathrm{D}_{\left(\eta_{1}, \eta_{2}\right)}\left(g_{x}(r) \circ \phi_{i}\right) \circ \mathrm{D} \phi_{i}^{-1} \text {. }
$$

By straigthforward computations we obtain that $\mathrm{D}_{\left(\eta_{1}, \eta_{2}\right)}\left(g_{x}(r) \circ \phi_{i}\right)=0$ for all $r$ [21. Thus by differentiation with respect to $r$ we prove that $g_{x}(r, y)$ and all its Gâteaux derivatives behaves as $|x-y|^{2}$ when $x-y \rightarrow 0$. 


\section{Shape differentiability of surface differential operators, application to hypersingular boundary integral operators}

Many classical hypersingular boundary integral operators can be expressed as compositions of boundary integral operators with pseudo-homogeneous weakly singular kernels and of surface differential operators. Such representations are often used in the numerical implementation of hypersingular boundary integral operators. Here we use these representations to study the shape derivatives of hypersingular boundary integral operators. To this end, in addition to the shape derivatives of the weakly singular integral boundary integral operators as studied in Section 4, we need to determine the Gâteaux derivatives with respect to deformations of the surface differential operators acting between Sobolev spaces: The tangential gradient is linear and continuous from $H^{t+1}(\Gamma)$ to $\mathbf{H}^{t}(\Gamma)$, the surface divergence is linear and continuous from $\mathbf{H}^{t+1}(\Gamma)$ to $H^{t}(\Gamma)$.

Example 5.1. (Acoustic hypersingular kernel) Let $\kappa \in \mathbb{C}$ with $\operatorname{Im}(\kappa) \geq 0$ and $d=3$. The hypersingular kernel is the normal derivative of the double layer kernel. We have

$$
\begin{aligned}
& \frac{\partial}{\partial \boldsymbol{n}(x)} \frac{\partial}{\partial \boldsymbol{n}(y)} G_{a}(\kappa, x-y) \\
& \quad=-\boldsymbol{n}(x) \cdot \boldsymbol{n}(y) \Delta G_{a}(\kappa, x-y)+\boldsymbol{n}(x) \cdot \operatorname{curl}^{x}\left(\nabla^{y} G_{a}(\kappa, x-y) \wedge \boldsymbol{n}(y)\right) .
\end{aligned}
$$

When $d=2$, for a scalar function $\varphi$ the term $-\nabla \varphi \wedge \boldsymbol{n}$ is the arc-length derivative $\frac{d \varphi}{d s}$. Using integration by parts with respect to the variable $y$ and that for a scalar function $v$ and a vector $\vec{a} \in \mathbb{R}^{d}$ it holds $\boldsymbol{n} \cdot \operatorname{curl}(v \vec{a})=$ $-(\nabla v \wedge \boldsymbol{n}) \cdot \vec{a}$ we obtain for a scalar density $u$

$$
\begin{aligned}
\int_{\Gamma} \boldsymbol{n}(x) \cdot \operatorname{curl}^{x} & \left(\nabla^{y}\left(G_{a}(\kappa, x-y)\right) \wedge \boldsymbol{n}(y)\right) u(y) d s(y) \\
= & -\int_{\Gamma}\left(\nabla^{x}\left(G_{a}(\kappa, x-y)\right) \wedge \boldsymbol{n}(x)\right) \cdot\left(\nabla^{y}(u(y)) \wedge \boldsymbol{n}(y)\right) d s(y) .
\end{aligned}
$$

Finally we have

$$
\begin{aligned}
\int_{\Gamma} \frac{\partial}{\partial \boldsymbol{n}(x)} \frac{\partial}{\partial \boldsymbol{n}(y)} & G_{a}(\kappa, x-y) u(y) d s(y) \\
= & \kappa^{2} \int_{\Gamma} G_{a}(\kappa, x-y) u(y)(\boldsymbol{n}(x) \cdot \boldsymbol{n}(y)) d s(y) \\
& -\int_{\Gamma}\left(\nabla_{\Gamma}^{x} G_{a}(\kappa, x-y) \wedge \boldsymbol{n}(x)\right) \cdot\left(\nabla_{\Gamma} u(y) \wedge \boldsymbol{n}(y)\right) d s(y) .
\end{aligned}
$$

A similar technique can be applied to the elastic hypersingular boundary integral operator using integration by part and Günter's tangential derivatives (see [11, 16]).

Lemma 5.2. Let $d=3$ and $\Gamma$ be a closed orientable surface in $\mathbb{R}^{3}$. The tangential Günter derivative denoted by $\mathcal{M}$ is defined for a vector function 
$\boldsymbol{v} \in \mathscr{C}^{1}\left(\Gamma, \mathbb{C}^{3}\right)$ by

$$
\mathcal{M} \boldsymbol{v}=\left(\nabla \boldsymbol{v}-(\operatorname{div} \boldsymbol{v}) \cdot \mathrm{I}_{\mathbb{R}^{3}}\right) \boldsymbol{n}=\frac{\partial}{\partial \boldsymbol{n}} \boldsymbol{v}-(\operatorname{div} \boldsymbol{v}) \boldsymbol{n}+\boldsymbol{n} \wedge \operatorname{curl} \boldsymbol{v}
$$

(i) We set $\boldsymbol{n}=\left(n_{k}\right)_{1 \leq k \leq 3}$ and $\mathcal{M}_{y}=\left(m_{j k}\right)_{1 \leq j, k \leq 3}$. We have

$$
m_{j k}=n_{k}(y) \frac{\partial}{\partial y_{j}}-n_{j}(y) \frac{\partial}{\partial y_{k}}=-m_{k j} .
$$

(ii) For any scalar functions $u, \tilde{u}$ in $\mathscr{C}^{1}(\Gamma, \mathbb{C})$ and vector functions $\boldsymbol{v}, \tilde{\boldsymbol{v}}$ in $\mathscr{C}^{1}\left(\Gamma, \mathbb{C}^{3}\right)$ there holds the Stokes formula

$$
\int_{\Gamma}\left(m_{j k} u\right) \cdot \tilde{u} d s=-\int_{\Gamma} u \cdot\left(m_{j k} \tilde{u}\right) d s \text { and } \int_{\Gamma}(\mathcal{M} \boldsymbol{v}) \cdot \tilde{\boldsymbol{v}} d s=+\int_{\Gamma} \boldsymbol{v} \cdot(\mathcal{M} \tilde{\boldsymbol{v}}) d s .
$$

Example 5.3. (Elastic hypersingular kernel) Let $\omega \in \mathbb{R}$ and $d=3$. Denote by $\rho, \mu$ and $\lambda$ the density and Lamé's constants. The hypersingular kernel is defined by

$$
H(x, y)=T_{x}\left(T_{y} G_{e}(\kappa, x-y)\right)^{\top}
$$

where $G_{e}$ is the fundamental solution of the Navier equation and $T$ is the traction operator defined in Example 2.5. First of all we rewrite the operator $T \boldsymbol{u}$ as

$$
T \boldsymbol{u}=2 \mu \mathcal{M} \boldsymbol{u}+(\lambda+2 \mu)(\operatorname{div} \boldsymbol{u}) \boldsymbol{n}-\mu \boldsymbol{n} \wedge \operatorname{curl} \boldsymbol{u} .
$$

Then we apply the operator $T_{y}$ in the form (5.2) to the tensor $G_{e}\left(\kappa_{s}, \kappa_{p}, x-y\right)$. It follows

$$
\begin{aligned}
& \left(T_{y} G_{e}\left(\kappa_{s}, \kappa_{p}, x-y\right)\right)^{\top} \\
& =2 \mu\left(\mathcal{M}_{y} G_{e}(\kappa, x-y)\right)^{\top}-\left(\boldsymbol{n}(y) \wedge \operatorname{curl}_{y} G_{a}\left(\kappa_{s}, x-y\right) \mathrm{I}_{\mathbb{R}^{3}}\right)^{\top} \\
& +\frac{(\lambda+2 \mu)}{\mu}\left(\boldsymbol{n}(y) \cdot \operatorname{div}_{y} G_{e}\left(\kappa_{s}, \kappa_{p}, x-y\right)\right)^{\top} .
\end{aligned}
$$

$$
\begin{array}{r}
\operatorname{div}_{y} G_{e}\left(\kappa_{s}, \kappa_{p}, x-y\right) \\
=\left(\nabla_{y} G_{a}\left(\kappa_{s}, x-y\right)\right)^{\top}+\frac{1}{\kappa_{s}^{2}} \nabla_{y}^{\top} \Delta_{y}\left(G_{a}\left(\kappa_{s}, x-y\right)-G_{a}\left(\kappa_{p}, x-y\right)\right) \\
=\frac{\kappa_{p}^{2}}{\kappa_{s}^{2}}\left(\nabla_{y} G_{a}\left(\kappa_{p}, x-y\right)\right)^{\top}
\end{array}
$$

$\boldsymbol{n}(y) \wedge \operatorname{curl}_{y} G_{a}\left(\kappa_{s}, x-y\right) \mathrm{I}_{\mathbb{R}^{3}}=\left(\mathcal{M}_{y}-\frac{\partial}{\partial \boldsymbol{n}(y)}+\boldsymbol{n}(y) \cdot \operatorname{div} y\right) G_{a}\left(\kappa_{s}, x-y\right) \mathrm{I}_{\mathbb{R}^{3}}$

In virtue of the property $(i)$ in Lemma 5.2 we can write

$$
\begin{aligned}
\left(\boldsymbol{n}(y) \wedge \operatorname{curl}_{y} G_{a}\left(\kappa_{s}, x-y\right) \mathrm{I}_{\mathbb{R}^{3}}\right)^{\top}= & \left(-\mathcal{M}_{y}-\frac{\partial}{\partial \boldsymbol{n}(y)}\right) G_{a}\left(\kappa_{s}, x-y\right) \mathrm{I}_{\mathbb{R}^{3}} \\
& +\left(\boldsymbol{n}(y) \cdot \nabla_{y}^{\top} G_{a}\left(\kappa_{s}, x-y\right)\right)^{\top}
\end{aligned}
$$


Collecting the equalities we obtain

$$
\begin{aligned}
& \left(T_{y} G_{e}\left(\kappa_{s}, \kappa_{p}, x-y\right)\right)^{\top} \\
& =2 \mu\left(\mathcal{M}_{y} G_{e}(\kappa, x-y)\right)^{\top}+\left(\frac{\partial}{\partial \boldsymbol{n}(y)}+\mathcal{M}_{y}\right) G_{a}\left(\kappa_{s}, x-y\right) \mathrm{I}_{\mathbb{R}^{3}} \\
& +\nabla_{y}\left(G_{a}\left(\kappa_{p}, x-y\right)-G_{a}\left(\kappa_{s}, x-y\right)\right) \cdot \boldsymbol{n}(y)^{\top} .
\end{aligned}
$$

By integration by part and using the properties $(i)$ and $(i i)$ of Lemma 5.2 we obtain that

$$
\begin{gathered}
\int_{\Gamma}\left(T_{y} G_{e}\left(\kappa_{s}, \kappa_{p}, x-y\right)\right)^{\top} \boldsymbol{u}(y) d s(y)=2 \mu \int_{\Gamma} G_{e}\left(\kappa_{s}, \kappa_{p}, x-y\right) \mathcal{M}_{y} \boldsymbol{u}(y) d s(y) \\
-\int_{\Gamma} G_{a}\left(\kappa_{s}, x-y\right) \mathcal{M}_{y} \boldsymbol{u}(y) d s(y)+\int_{\Gamma} \frac{\partial}{\partial \boldsymbol{n}(y)} G_{a}\left(\kappa_{s}, x-y\right) \boldsymbol{u}(y) d s(y) \\
+\int_{\Gamma} \nabla_{y}\left(G_{a}\left(\kappa_{p}, x-y\right)-G_{a}\left(\kappa_{s}, x-y\right)\right)(\boldsymbol{n}(y) \cdot \boldsymbol{u}(y)) d s(y)
\end{gathered}
$$

The kernel of the last term in the right hand side is pseudo-homogeneous of class -2 . Thus $T_{x}$ applied to this term yields a pseudo-homogeneous kernel of class -1 . Similarly to $\left(T_{y} G_{e}\left(\kappa_{s}, \kappa_{p}, x-y\right)\right)^{\top}$, the kernel $T_{x} G_{e}\left(\kappa_{s}, \kappa_{p}, x-y\right)$ can be rewritten in terms of products of weakly singular kernels and the Günter derivative $\mathcal{M}_{x}$. Now we apply the operator $T_{x}$ to the kernels of the second and third terms on the right hand side in the form

$$
T_{x} \boldsymbol{u}=(\lambda+\mu) \boldsymbol{n}\left(\operatorname{div}_{x} \boldsymbol{u}\right)+\mu\left(\frac{\partial}{\partial \boldsymbol{n}(x)}+\mathcal{M}_{x}\right) \boldsymbol{u}
$$

We obtain

$$
\begin{aligned}
& T_{x}\left\{\frac{\partial}{\partial \boldsymbol{n}(y)} G_{a}\left(\kappa_{s}, x-y\right) \cdot \mathrm{I}_{R^{3}}\right\}=\mu \frac{\partial^{2}}{\partial \boldsymbol{n}(x) \partial \boldsymbol{n}(y)} G_{a}\left(\kappa_{s}, x-y\right) \cdot \mathrm{I}_{\mathbb{R}^{3}} \\
& +\mu \mathcal{M}_{x}\left(\frac{\partial}{\partial \boldsymbol{n}(y)} G_{a}\left(\kappa_{s}, x-y\right) \cdot \mathrm{I}_{\mathbb{R}^{3}}\right)+(\lambda+\mu) \boldsymbol{n}(x) \cdot \nabla_{x}^{\top} \frac{\partial}{\partial \boldsymbol{n}(y)} G_{a}\left(\kappa_{s}, x-y\right) \\
& -T_{x}\left\{G_{a}\left(\kappa_{s}, x-y\right) \cdot \mathrm{I}_{R^{3}}\right\}=-\mu \frac{\partial}{\partial \boldsymbol{n}(x)} G_{a}\left(\kappa_{s}, x-y\right) \cdot \mathrm{I}_{\mathbb{R}^{3}} \\
& \quad-\mu \mathcal{M}_{x}\left(G_{a}\left(\kappa_{s}, x-y\right) \cdot \mathrm{I}_{\mathbb{R}^{3}}\right)-(\lambda+\mu) \boldsymbol{n}(x) \cdot \nabla_{x}^{\top} G_{a}\left(\kappa_{s}, x-y\right)
\end{aligned}
$$

We use the equality

$$
\nabla_{x} \frac{\partial}{\partial \boldsymbol{n}(y)} G_{a}\left(\kappa_{s}, x-y\right)=\mathcal{M}_{y} \nabla_{x} G_{a}\left(\kappa_{s}, x-y\right)-\boldsymbol{n}(y) \Delta_{y} G_{a}\left(\kappa_{s}, x-y\right)
$$


and Lemma 5.2 to show that (see [1] pp. 52)

$$
\begin{aligned}
\int_{\Gamma} \boldsymbol{n}(x) \cdot \nabla_{x}^{\top} & \frac{\partial}{\partial \boldsymbol{n}(y)} G_{a}\left(\kappa_{s}, x-y\right) \boldsymbol{u}(y) d s(y) \\
& -\int_{\Gamma} \boldsymbol{n}(x) \cdot \nabla_{x}^{\top} G_{a}\left(\kappa_{s}, x-y\right) \mathcal{M}_{y} \boldsymbol{u}(y) d s(y) \\
& =\kappa_{s}^{2} \boldsymbol{n}(x) \int_{\Gamma} G_{a}\left(\kappa_{s}, x-y\right)(\boldsymbol{n}(y) \cdot \boldsymbol{u}(y)) d s(y) .
\end{aligned}
$$

Finally we have

$$
\begin{gathered}
\int_{\Gamma} T_{x}\left(T_{y} G_{e}\left(\kappa_{s}, \kappa_{p}, x-y\right)\right)^{\top} \boldsymbol{u}(y) d s(y) \\
=2 \mu \int_{\Gamma}\left[T_{x} G_{e}\left(\kappa_{s}, \kappa_{p}, x-y\right)\right] \mathcal{M}_{y} \boldsymbol{u}(y) d s(y) \\
+\mu \int_{\Gamma} \frac{\partial^{2}}{\partial \boldsymbol{n}(x) \partial \boldsymbol{n}(y)} G_{a}\left(\kappa_{s}, x-y\right) \boldsymbol{u}(y) d s(y) \\
\quad-\mu \int_{\Gamma} \frac{\partial}{\partial \boldsymbol{n}(x)} G_{a}\left(\kappa_{s}, x-y\right) \mathcal{M}_{y} \boldsymbol{u}(y) d s(y) \\
-\mu \mathcal{M}_{x} \int_{\Gamma} G_{a}\left(\kappa_{s}, x-y\right) \mathcal{M}_{y} \boldsymbol{u}(y) d s(y)+\mu \mathcal{M}_{x} \int_{\Gamma} \frac{\partial}{\partial \boldsymbol{n}(y)} G_{a}\left(\kappa_{s}, x-y\right) \boldsymbol{u}(y) d s(y) \\
+\int_{\Gamma} T_{x} \nabla_{y}\left(G_{a}\left(\kappa_{p}, x-y\right)-G_{a}\left(\kappa_{s}, x-y\right)\right)(\boldsymbol{n}(y) \cdot \boldsymbol{u}(y)) d s(y) \\
+\kappa_{s}^{2}(\lambda+\mu) \boldsymbol{n}(x) \int_{\Gamma} G_{a}\left(\kappa_{s}, x-y\right)(\boldsymbol{n}(y) \cdot \boldsymbol{u}(y)) d s(y) .
\end{gathered}
$$

We see that the boundary integral operator with either the acoustic hypersingular kernel or the elastic hypersingular kernel are operators of order +1 on the Sobolev spaces $H^{t}(\Gamma)$ for $t \in \mathbb{R}$. Using the integral representations above, the differentiability properties of these operators can be deduced from the knowledge of the differentiability properties of the surface differential operators. Following the same pullback procedure as in Section 4 , the analysis of the hypersingular integral operators is finally reduced to the analysis of the mappings

$$
\begin{aligned}
r & \mapsto \tau_{r} \nabla_{\Gamma_{r}} \tau_{r}^{-1} \\
r & \mapsto \tau_{r} \operatorname{div}_{\Gamma_{r}} \tau_{r}^{-1} .
\end{aligned}
$$

Indeed, the Günter derivative can be rewritten in terms of these two differential operators:

$$
\mathcal{M} \boldsymbol{v}=\left(\nabla_{\Gamma} \boldsymbol{v}-\left(\operatorname{div}_{\Gamma} \boldsymbol{v}\right) \cdot \mathrm{I}_{\mathbb{R}^{3}}\right) \boldsymbol{n} .
$$

The results are established in the following theorems.

Theorem 5.4. The mapping

$$
\begin{array}{cccc}
\mathcal{G}: \quad B^{\infty}(0, \varepsilon) & \rightarrow & \mathscr{L}\left(H^{t+1}(\Gamma), \mathbf{H}^{t}(\Gamma)\right) \\
r & \mapsto & \tau_{r} \nabla_{\Gamma_{r}} \tau_{r}^{-1}
\end{array}
$$


is $\mathscr{C}^{\infty}$-Gâteaux differentiable and its first derivative at $r_{0}$ is defined for $\xi \in$ $\mathscr{C}^{\infty}\left(\Gamma, \mathbb{R}^{d}\right)$ by

$$
d \mathcal{G}\left[r_{0}, \xi\right] u=-\left[\mathcal{G}\left(r_{0}\right) \xi\right] \mathcal{G}\left(r_{0}\right) u+\left(\mathcal{G}\left(r_{0}\right) u \cdot\left[\mathcal{G}\left(r_{0}\right) \xi\right] \mathcal{N}\left(r_{0}\right)\right) \mathcal{N}\left(r_{0}\right) .
$$

Remark 5.5. Note that we can write $d \mathcal{N}\left[r_{0}, \xi\right]=-\left[\mathcal{G}\left(r_{0}\right) \xi\right] \mathcal{N}\left(r_{0}\right)$. Since the first derivatives of $\mathcal{N}$ and $\mathcal{G}$ are expressed in terms of $\mathcal{N}$ and $\mathcal{G}$, we can obtain the Gâteaux derivatives of all orders recursively.

Proof. In accordance with the Definition (4.6) and Lemma 4.3 to prove the $\mathscr{C}^{\infty}$-Gâteaux differentiability of $\mathcal{G}$ we have to prove the $\mathscr{C}^{\infty}$-Gâteaux differentiability of the mapping

$$
f: B^{\infty}(0, \varepsilon) \ni r \mapsto\left\{u \mapsto \tau_{r}\left(\widetilde{\nabla \tau_{r}^{-1} u}\right)_{\left.\right|_{\Gamma}}\right\} \in \mathscr{L}\left(H^{t+1}(\Gamma), \mathbf{H}^{t}(\Gamma)\right) .
$$

For $x \in \Gamma$, we have

$$
\begin{aligned}
\tau_{r}\left(\widetilde{\nabla \tau_{r}^{-1} u}\right)_{\left.\right|_{\Gamma_{r}}}(x)=\nabla\left(\widetilde{u} \circ(\mathrm{I}+r)^{-1}\right)_{\left.\right|_{\Gamma_{r}}}(x+r(x)) \\
=(\mathrm{I}+\mathrm{D} r)_{\left.\right|_{\Gamma_{r}} ^{-1}}(x+r(x)) \circ \nabla \widetilde{u}_{\left.\right|_{\Gamma}}(x),
\end{aligned}
$$

and

$$
(\mathrm{I}+\mathrm{D} r)_{\left.\right|_{\Gamma_{r}}}^{-1}(x+r(x))=\left[(\mathrm{I}+\mathrm{D} r)_{\left.\right|_{\Gamma}}(x)\right]^{-1} .
$$

The mapping $g: B^{\infty}(0, \varepsilon) \ni r \mapsto(\mathrm{I}+\mathrm{D} r)_{\left.\right|_{\Gamma}} \in \mathscr{C}^{\infty}(\Gamma)$ is continuous, and $\mathscr{C}^{\infty}$-Gâteaux differentiable. Its first derivative is $d g[0, \xi]=[\mathrm{D} \xi]_{\left.\right|_{\Gamma}}$ and its higher order derivatives vanish. One can easily see that the mapping $h: r \in$ $B_{\epsilon}^{\infty} \mapsto\left\{x \mapsto[g(r)]^{-1}(x)\right\} \in \mathscr{C}^{\infty}(\Gamma)$ is also $\mathscr{C}^{\infty}$ Gâteaux-differentiable and that we have at $r_{0}$ and in the direction $\xi$ :

$$
d h\left[r_{0}, \xi\right]=-h\left(r_{0}\right) \circ d g\left[r_{0}, \xi\right] \circ h\left(r_{0}\right)=-h\left(r_{0}\right) \circ[\mathrm{D} \xi]_{\left.\right|_{\Gamma}} \circ h\left(r_{0}\right) .
$$

and

$d^{n} h\left[r_{0}, \xi_{1}, \ldots, \xi_{n}\right]=(-1)^{n} \sum_{s \in \mathscr{S}_{n}}\left(\mathrm{I}+\mathrm{D} r_{0}\right)^{-1} \circ\left[\tau_{r_{0}} \mathrm{D} \tau_{r_{0}}^{-1} \xi_{s(1)}\right] \circ \ldots \circ\left[\tau_{r_{0}} \mathrm{D} \tau_{r_{0}}^{-1} \xi_{s(n)}\right]$

where $\mathscr{S}_{n}$ is the permutation group of $\{1, \ldots, n\}$. Finally we obtain the $\mathscr{C}^{\infty}$ Gâteaux differentiability of $f$ and we have

$$
d f\left[r_{0}, \xi\right] u=-\left[f\left(r_{0}\right) \xi\right] f\left(r_{0}\right) u .
$$

Notice that this result can also be justified by using commutators : for example at $r=0$ in the direction, we have

$$
\frac{\partial}{\partial r}\left(\tau_{r} \nabla \tau_{r}^{-1} u\right)[0, \xi]=\frac{\partial}{\partial \xi}(\nabla u)-\nabla \frac{\partial}{\partial \xi} u=-[\nabla \xi] \nabla \boldsymbol{u}
$$

where $\frac{\partial}{\partial \xi}=\xi \cdot \nabla$.

To obtain the expression of the first derivative of $\mathcal{G}$ we have to differentiate the following expression:

$$
\begin{aligned}
\mathcal{G}(r) u & =\left(\tau_{r} \nabla_{\Gamma_{r}} \tau_{r}^{-1} u\right)=\tau_{r} \nabla\left(\widetilde{\tau_{r}^{-1} u}\right)-\left(\tau_{r} \boldsymbol{n}_{r} \cdot\left(\tau_{r} \nabla\left(\widetilde{\tau_{r}^{-1} u}\right)\right)\right) \tau_{r} \boldsymbol{n}_{r} \\
& =f(r) u-(f(r) u \cdot \mathcal{N}(r)) \mathcal{N}(r) .
\end{aligned}
$$


By Lemma 4.3 and the chain and product rules we have

$$
\begin{aligned}
d \mathcal{G}\left[r_{0}, \xi\right] & =-\left[f\left(r_{0}\right) \xi\right] f\left(r_{0}\right) u+\left(\left[f\left(r_{0}\right) \xi\right] f\left(r_{0}\right) u \cdot \mathcal{N}\left(r_{0}\right)\right) \mathcal{N}\left(r_{0}\right) \\
& +\left(f\left(r_{0}\right) u \cdot\left[\mathcal{G}\left(r_{0}\right) \xi\right] \mathcal{N}\left(r_{0}\right)\right) \mathcal{N}\left(r_{0}\right)+\left(f\left(r_{0}\right) u \cdot \mathcal{N}\left(r_{0}\right)\right)\left[G\left(r_{0}\right) \xi\right] \mathcal{N}\left(r_{0}\right)
\end{aligned}
$$

Combining the first two terms in the right hand side, we get

$$
\begin{aligned}
d \mathcal{G}\left[r_{0}, \xi\right]=- & {\left[\mathcal{G}\left(r_{0}\right) \xi\right] f\left(r_{0}\right) u+\left(f\left(r_{0}\right) u \cdot \mathcal{N}\left(r_{0}\right)\right)\left[\mathcal{G}\left(r_{0}\right) \xi\right] \mathcal{N}\left(r_{0}\right) } \\
& +\left(f\left(r_{0}\right) u \cdot\left[\mathcal{G}\left(r_{0}\right) \xi\right] \mathcal{N}\left(r_{0}\right)\right) \mathcal{N}\left(r_{0}\right) \\
=- & {\left[\mathcal{G}\left(r_{0}\right) \xi\right] \mathcal{G}\left(r_{0}\right) u+\left(f\left(r_{0}\right) u \cdot\left[\mathcal{G}\left(r_{0}\right) \xi\right] \mathcal{N}\left(r_{0}\right)\right) \mathcal{N}\left(r_{0}\right) . }
\end{aligned}
$$

To conclude, it suffices to note that

$$
\left(f\left(r_{0}\right) u \cdot\left[\mathcal{G}\left(r_{0}\right) \xi\right] \mathcal{N}\left(r_{0}\right)\right)=\left(\mathcal{G}\left(r_{0}\right) u \cdot\left[\mathcal{G}\left(r_{0}\right) \xi\right] \mathcal{N}\left(r_{0}\right)\right) .
$$

Theorem 5.6. The mapping

$$
\begin{array}{cccc}
\mathcal{D}: B^{\infty}(0, \varepsilon) & \rightarrow & \mathscr{L}\left(\mathbf{H}^{t+1}(\Gamma), H^{t}(\Gamma)\right) \\
r & \mapsto & \tau_{r} \operatorname{div}_{\Gamma_{r}} \tau_{r}^{-1}
\end{array}
$$

is $\mathscr{C}^{\infty}$-Gâteaux differentiable and its first derivative at $r_{0}$ is defined for $\xi \in$ $\mathscr{C}^{\infty}\left(\Gamma, \mathbb{R}^{d}\right)$ by

$$
d \mathcal{D}\left[r_{0}, \xi\right] \boldsymbol{u}=-\operatorname{Trace}\left(\left[\mathcal{G}\left(r_{0}\right) \xi\right]\left[\mathcal{G}\left(r_{0}\right) \boldsymbol{u}\right]\right)+\left(\left[\mathcal{G}\left(r_{0}\right) \boldsymbol{u}\right] \mathcal{N}\left(r_{0}\right) \cdot\left[\mathcal{G}\left(r_{0}\right) \xi\right] \mathcal{N}\left(r_{0}\right)\right) .
$$

Proof. For $\boldsymbol{u} \in \mathbf{H}^{t+1}(\Gamma)$ we have $\mathcal{D}(r) \boldsymbol{u}=\operatorname{Trace}([\mathcal{G}(r) \boldsymbol{u}])$. Then we use the differentiation rules.

Remark 5.7. (i) Since the first derivative of $\mathcal{D}$ is composed of $\mathcal{G}$ and $\mathcal{N}$ and the first derivative of $\mathcal{J}$ is composed of $\mathcal{J}$ and $\mathcal{D}$, we can obtain an expression of higher order derivatives of the Jacobian recursively.

(ii) Denoting by $\mathcal{M}_{\Gamma_{r}}$ the tangential Günter derivative on $\Gamma_{r}$, the formulas (\#) in section 4 can be rewritten as

$$
\left\{\begin{aligned}
\mathcal{W}\left(r_{0}\right) & =J_{r_{0}}\left(\tau_{r_{0}} \boldsymbol{n}_{r_{0}}\right), \\
\frac{\partial \mathcal{W}}{\partial r}\left[r_{0}, \xi\right] & =-J_{r_{0}}\left(\tau_{r_{0}} \mathcal{M}_{\Gamma_{r_{0}}}\left(\tau_{r_{0}}^{-1} \xi\right)\right), \\
\frac{\partial^{m} \mathcal{W}}{\partial r^{m}}\left[r_{0}, \xi\right] & \equiv 0 \text { for all } m \geq d .
\end{aligned}\right.
$$

Remark 5.8. (Electromagnetic hypersingular kernel) Let $\kappa \in \mathbb{C}$ with $\operatorname{Im}(\kappa) \geq$ 0 and $d=3$. The electromagnetic hypersingular operator is defined for a tangential density $\mathbf{j} \in \mathbf{T} \mathbf{H}^{t}(\Gamma)$ by

$$
C_{\kappa} \mathbf{j}(x)=-\frac{1}{\kappa} \int_{\Gamma} \boldsymbol{n}(x) \wedge\left(\operatorname{curl}^{x} \operatorname{curl}^{x}\left(G_{a}(\kappa, x-y) \mathbf{j}(y)\right)\right) d s(y) .
$$

Using the identity curl curl $=-\Delta+\nabla$ div we have

$$
\begin{aligned}
C_{\kappa} \mathbf{j}(x)=-\boldsymbol{n}(x) & \wedge \int_{\Gamma}\left(\kappa G_{a}(\kappa, x-y) \cdot \mathrm{I}_{\mathbb{R}^{3}}\right. \\
+ & \left.\frac{1}{\kappa} \nabla_{\Gamma}^{x} G_{a}(\kappa, x-y) \operatorname{div}_{\Gamma}\right) \mathbf{j}(y) d s(y) .
\end{aligned}
$$


This is the operator of the electric field integral equation in electromagnetism. The operator $C_{\kappa}$ is a priori an operator of order +1 on the space of tangential vector functions $\mathbf{T H}^{t}(\Gamma)$, but it is well known that this operator is a bounded Fredholm operator on the space of tangential vector fields of mixed regularity $\mathbf{T} \mathbf{H}^{-\frac{1}{2}}\left(\operatorname{div}_{\Gamma}, \Gamma\right)$, the set of tangential vector fields whose components are in the Sobolev space $H^{-\frac{1}{2}}(\Gamma)$ and whose surface divergence is in $H^{-\frac{1}{2}}(\Gamma)$. Therefore it is desirable to study the shape differentiability of this operator defined on the shape dependent space $\mathbf{T H}^{-\frac{1}{2}}\left(\operatorname{div}_{\Gamma}, \Gamma\right)$. For this, the tools presented above are not directly applicable. It is the purpose of the second part [3] of our paper to present an alternative strategy using the Helmholtz decomposition of the space $\mathbf{T} \mathbf{H}^{-\frac{1}{2}}\left(\operatorname{div}_{\Gamma}, \Gamma\right)$.

\section{References}

[1] A. Charalambopoulos, On the Fréchet differentiability of boundary integral operators in the inverse elastic scattering problem, Inverse Problems, 11 (1995), pp. 1137-1161.

[2] D. Colton And R. Kress, Inverse acoustic and electromagnetic scattering theory, vol. 93 of Applied Mathematical Sciences, Springer-Verlag, Berlin, second ed., 1998.

[3] M. Costabel And F. Le LouËr, Shape derivatives of boundary integral operators in electromagnetic scattering. Part II: Application to scattering by a homogeneous dielectric obstacle, (2011).

[4] A. DE La Bourdonnaye, Décomposition de $H_{\mathrm{div}}^{-1 / 2}(\Gamma)$ et nature de l'opérateur de Steklov-Poincaré du problème extérieur de l'électromagnétisme, C. R. Acad. Sci. Paris Sér. I Math., 316 (1993), pp. 369-372.

[5] M. C. Delfour And J.-P. Zolésio, Shapes and geometries, vol. 4 of Advances in Design and Control, Society for Industrial and Applied Mathematics (SIAM), Philadelphia, PA, 2001. Analysis, differential calculus, and optimization.

[6] M. C. Delfour And J.-P. ZolÉsio, Tangential calculus and shape derivatives, vol. 216 of Lecture Notes in Pure and Appl. Math., Dekker, New York, 2001.

[7] G. I. ÈsKIN, Boundary value problems for elliptic pseudodifferential equations, Moscow, 1973.

[8] J. Hadamard, Sur quelques questions du calcul des variations, Ann. Sci. École Norm. Sup. (3), 24 (1907), pp. 203-231.

[9] A. Henrot And M. Pierre, Variation et optimisation de formes, vol. 48 of Mathématiques \& Applications (Berlin) [Mathematics \& Applications], Springer, Berlin, 2005. Une analyse géométrique. [A geometric analysis].

[10] L. HÖRMANDER, The analysis of linear partial differential operators. III, Classics in Mathematics, Springer, Berlin, 2007. Pseudo-differential operators, Reprint of the 1994 edition.

[11] G. C. Hsiao And W. L. Wendland, Boundary integral equations, vol. 164 of Applied Mathematical Sciences, Springer-Verlag, Berlin, 2008.

[12] O. IVANYSHYN, Shape reconstruction of acoustic obstacles from the modulus of the far field pattern, Inverse Probl. Imaging, 1 (2007), pp. 609-622. 
[13] O. IVANyShyn AND R. KREss, Nonlinear integral equations in inverse obstacle scattering, (2006), pp. 39-50.

[14] J.-L. JouRné, Calderón-Zygmund operators, pseudodifferential operators and the Cauchy integral of Calderón, vol. 994 of Lecture Notes in Mathematics, Springer-Verlag, Berlin, 1983.

[15] R. KRess And W. Rundell, Nonlinear integral equations and the iterative solution for an inverse boundary value problem, Inverse Problems, 21 (2005), pp. 1207-1223.

[16] V. D. Kupradze, T. G. Gegelia, M. O. BasheleǏshvili, and T. V. BuRChUlADZE, Three-dimensional problems of the mathematical theory of elasticity and thermoelasticity, vol. 25 of North-Holland Series in Applied Mathematics and Mechanics, North-Holland Publishing Co., Amsterdam, russian ed., 1979. Edited by V. D. Kupradze.

[17] F. LE LouËR, Optimisation de formes d'antennes lentilles intégrées aux ondes millimétriques, PhD in Numérical Analysis, Université de Rennes 1, 2009. http://tel.archives-ouvertes.fr/tel-00421863/fr/.

[18] G. Leugering, A. A. Novotny, G. P. Menzala, and J. Sokołowski, On shape optimization for an evolution coupled system, Appl. Math. Optim. 64, (2011), pp. 441-466.

[19] Y. Meyer, Ondelettes et opérateurs. II, Actualités Mathématiques. [Current Mathematical Topics], Hermann, Paris, 1990. Opérateurs de CalderónZygmund. [Calderón-Zygmund operators].

[20] J.-C. NÉDÉLEC, Acoustic and electromagnetic equations, vol. 144 of Applied Mathematical Sciences, Springer-Verlag, New York, 2001. Integral representations for harmonic problems.

[21] R. Pоттнаst, Fréchet differentiability of boundary integral operators in inverse acoustic scattering, Inverse Problems, 10 (1994), pp. 431-447.

[22] — Fréchet differentiability of the solution to the acoustic Neumann scattering problem with respect to the domain, J. Inverse Ill-Posed Probl., 4 (1996), pp. $67-84$.

[23] J. T. Schwartz, Nonlinear functional analysis, Gordon and Breach Science Publishers, New York, 1969. Notes by H. Fattorini, R. Nirenberg and H. Porta, with an additional chapter by Hermann Karcher, Notes on Mathematics and its Applications.

[24] J. SokoŁowski AND J.-P. ZoLÉSIO, Introduction to shape optimization, vol. 16 of Springer Series in Computational Mathematics, Springer-Verlag, Berlin, 1992. Shape sensitivity analysis.

[25] M. E. TAYlor, Partial differential equations. I, vol. 115 of Applied Mathematical Sciences, Springer-Verlag, New York, 1996. Basic theory.

[26] - Tools for PDE, vol. 81 of Mathematical Surveys and Monographs, American Mathematical Society, Providence, RI, 2000. Pseudodifferential operators, paradifferential operators, and layer potentials.

Martin Costabel

IRMAR, Institut Mathématique, Université de Rennes 1, 35042 Rennes, France e-mail: martin.costabel@univ-rennes1.fr 
Frédérique Le Louër

Institut für Numerische und Andgewandte Mathematik, Universität Göttingen, 37083 Göttingen, Germany

e-mail: f.lelouer@math.uni-goettingen.de 